\title{
Backward SDEs with superquadratic growth
}

\author{
Freddy Delbaen, $\quad$ Ying $\mathrm{Hu}^{\dagger}$ and Xiaobo Bao ${ }^{\ddagger}$
}

February 19, 2009

\begin{abstract}
In this paper, we discuss the solvability of backward stochastic differential equations (BSDEs) with superquadratic generators. We first prove that given a superquadratic generator, there exists a bounded terminal value, such that the associated BSDE does not admit any bounded solution. On the other hand, we prove that if the superquadratic BSDE admits a bounded solution, then there exist infinitely many bounded solutions for this BSDE. Finally, we prove the existence of a solution for Markovian BSDEs where the terminal value is a bounded continuous function of a forward stochastic differential equation.
\end{abstract}

\section{Introduction.}

Since the pioneer works on BSDEs of Bismut [2] and Pardoux-Peng [13], lots of works have been done in this area and the original Lipschitz assumption on the generator, i.e., the function $g$ in the BSDE:

$$
Y_{t}=\xi-\int_{t}^{T} g\left(s, Y_{s}, Z_{s}\right) d s+\int_{t}^{T} Z_{s} d B_{s}, \quad 0 \leq t \leq T
$$

*Department of Mathematics, ETH Zürich, Switzerland. E-mail: delbaen@math.ethz.ch. Part of the research was done while this author was visiting Princeton, Vancouver and China. The hospitalities of Princeton University, UBC, Fudan University and Shandong University are greatly appreciated.

${ }^{\dagger}$ IRMAR, Université Rennes 1, 35042 RENNES Cedex, France. E-mail: ying.hu@univ-rennes1.fr.

${ }^{\ddagger}$ Department of Mathematics, ETH Zürich, Switzerland. E-mail: baoxb@math.ethz.ch. 
has been weakened in many situations. Let us recall that, in the previous BSDE, we are looking for a pair of processes $(Y, Z)$ which is required to be predictable with respect to the filtration generated by the Brownian motion $B$. One of the most important works in this direction is that of Kobylanski [12] concerning scalar-valued quadratic BSDEs with bounded terminal value. We should point out that quadratic BSDE means a BSDE whose generator has at most a quadratic growth with respect to the variable z. For these quadratic BSDEs, all the classical results, existence and uniqueness, comparison and stability of solutions, have been stated in [12] but with the restriction that the terminal conditions have to be bounded random variables. Recently, existence and uniqueness of solutions of quadratic BSDEs with unbounded terminal value were studied by Briand and $\mathrm{Hu}$ in [3, 4].

In this paper, we study the solvability of superquadratic BSDE (1.1) whose generator $g$ is superquadratic, i.e.,

$$
\varlimsup_{|z| \rightarrow+\infty} \frac{g(z)}{|z|^{2}}=\infty
$$

We shall study this BSDE with bounded terminal value. And in addition, we suppose that $g$ is a deterministic convex (or concave) function which is independent of $y$ with $g(0)=0$.

The first part of this paper shows the ill-posedness of these BSDEs. We first prove that given a superquadratic generator, there always exists a bounded terminal value, such that the associated BSDE does not admit any bounded solution. On the other hand, we prove that if the superquadratic BSDE admits a bounded solution, then there exist infinitely many bounded solutions for this BSDE. And finally, we show that the monotone stability, which plays a crucial role in quadratic BSDEs (see, e.g., [12, 3]), does not hold.

In the second part of this paper, we study BSDE (1.1) in the Markovian case, i.e., the terminal value

$$
\xi=\Phi\left(X_{T}^{t, x}\right)
$$

where the diffusion process $X$ is the solution to the SDE:

$$
X_{s}=x+\int_{t}^{s} b\left(r, X_{r}\right) d r+\int_{t}^{s} \sigma d B_{r}, \quad t \leq s \leq T .
$$

It is by now well-known (see, e.g., [14, 12, 4] ) that, if $g$ is Lipschitz or quadratic, 
there exists a link between the solution of (1.1) and that of the following PDE:

$$
\left\{\begin{array}{l}
u_{t}(t, x)+\frac{1}{2} \operatorname{trace}\left(\sigma \sigma^{T} u_{x x}(t, x)\right)+u_{x}(t, x) b(t, x)-g\left(-u_{x}(t, x) \sigma\right)=0, \\
u(T, x)=\Phi(x) .
\end{array}\right.
$$

This type of PDE (called viscous Hamilton-Jacobi equation) is already well studied when $\sigma$ is the identity and $g(z)=-|z|^{p}$, see, e.g., Gilding et al. [10] and Ben-Artzi et al. [1]. In particular, in [10, they established the existence and uniqueness of classical solution to this $\mathrm{PDE}$ when $\sigma$ is the identity.

We prove that in the Markovian case, the BSDE (1.1) admits a solution when $\Phi$ is bounded and continuous. Moreover, if we define

$$
u(t, x)=Y_{t}^{t, x}
$$

then $u$ is a continuous viscosity solution to PDE (1.3). We note that in our case, some kind of degeneracy of $\sigma$ is allowed, whereas in [10] and [1, they assumed that $\sigma$ is the identity.

A key idea to prove the existence in the Markovian case comes from the following a priori estimate of $Z$ :

$$
\left|Z_{t}\right| \leq c|| \Phi \|_{\infty}(T-t)^{-\frac{1}{2}}
$$

where $c>0$ is a constant. We prove this inequality by using a stochastic argument based on BMO martingales and Jensen's inequality. Note that Gilding et al. [10] proved the same type of a priori estimate for $u_{x}$ when $\sigma$ is identity, by use of Bernstein's method.

The paper is organized as follows: in the next section, we give some preliminaries about the connection between dynamic utility functions and BSDEs. Section 3 shows the ill-posedness in the general case. The last section is devoted to the proof of the existence of a solution in the Markovian case.

\section{Dynamic Utility Functions and Backward SDEs.}

Let $\left\{B_{t}, 0 \leq t \leq T\right\}$ be a $d$-dimensional standard Brownian motion defined on a probability space $(\Omega, \mathcal{F}, P)$. Let $\left\{\mathcal{F}_{t}, 0 \leq t \leq T\right\}$ be the natural filtration of $\left\{B_{t}, t \in\right.$ $[0, T]\}$, augmented by all $P$-null sets of $\mathcal{F}$. 
Before recalling the definition of dynamic utility functions, we need the following notations.

$$
\begin{aligned}
L^{\infty}\left(\mathcal{F}_{T}\right) & :=\left\{\xi: \text { bounded and } \mathcal{F}_{T} \text {-measurable random variable }\right\} \\
\mathcal{L}_{\mathcal{F}}^{2}\left(0, T ; \mathbb{R}^{m}\right) & :=\left\{\varphi: \mathbb{R}^{m} \text {-valued, }\left\{\mathcal{F}_{t}\right\}_{0 \leq t \leq T} \text {-predictable and } E\left[\int_{0}^{T}\left|\varphi_{t}\right|^{2} d t\right]<\infty\right\} .
\end{aligned}
$$

We identify random variables that are equal $P$ a.s.

Definition 2.1. We call a dynamic utility function with the Fatou property any family of operators, indexed by stopping times $\sigma$

$$
U_{\sigma}: L^{\infty}\left(\mathcal{F}_{T}\right) \rightarrow L^{\infty}\left(\mathcal{F}_{\sigma}\right)
$$

and satisfying:

- (A1) Positivity: $U_{\sigma}(0)=0, U_{\sigma}(\xi) \geq 0$ for all $\xi \geq 0$.

- (A2) Concavity: $U_{\sigma}(t \xi+(1-t) \eta) \geq t U_{\sigma}(\xi)+(1-t) U_{\sigma}(\eta)$, for all $t, 0 \leq t \leq$ 1 and all $\xi, \eta \in L^{\infty}$.

- (A3) Translability: $U_{\sigma}(\xi+a)=U_{\sigma}(\xi)+a$, for all $a \in L^{\infty}\left(\mathcal{F}_{\sigma}\right)$.

- (A4) Fatou property: Given a sequence $\left(\xi_{n}\right)_{n \geq 1}$, such that $\sup \left\|\xi_{n}\right\|_{\infty}<\infty$, then $\xi_{n} \downarrow \xi$ a.s. implies $U_{\sigma}(\xi)=\lim _{n \rightarrow \infty} U_{\sigma}\left(\xi_{n}\right)$ a.s.

For a lower semi-continuous convex function $f: R^{d} \rightarrow R_{+} \cup\{\infty\}$ such that $f(0)=0$ and for $\xi \in L^{\infty}\left(\mathcal{F}_{T}\right)$, we define

$$
U_{\sigma}(\xi)=\operatorname{ess.inf}\left\{E_{Q}\left[\xi+\int_{\sigma}^{T} f\left(q_{u}\right) d u \mid \mathcal{F}_{\sigma}\right] \mid Q \sim P\right\}
$$

where $\sigma \in[0, T]$ is a stopping time and the density process $E_{P}\left[\frac{d Q}{d P} \mid \mathcal{F}_{t}\right]=\mathcal{E}(q \cdot B)_{t}=$ $\exp \left(\int_{0}^{t} q_{u} d B_{u}-\frac{1}{2} \int_{0}^{t}\left|q_{u}\right|^{2} d u\right)$. It is easy to prove that $U$ is a dynamic utility function. As shown by Delbaen-Peng-Rosazza Gianin [7, $U$ is time consistent and all time consistent dynamic utility functions are of a similar form.

Set $C_{0}(Q)=E_{Q}\left[\int_{0}^{T} f\left(q_{u}\right) d u\right]$ and $\mathcal{P}=\{Q \mid Q \ll P\}$. The utility function $U_{0}$ can be defined by $\mathcal{P}$.

Lemma 2.1. For any $\xi \in L^{\infty}\left(\mathcal{F}_{T}\right)$,

$$
U_{0}(\xi)=\inf \left\{E_{Q}\left[\xi+\int_{0}^{T} f\left(q_{u}\right) d u\right] \mid Q \in \mathcal{P}\right\}
$$


Proof. For any $Q \in \mathcal{P}$ with $L_{t}=E_{P}\left[\frac{d Q}{d P} \mid \mathcal{F}_{t}\right]=\mathcal{E}(q \cdot B)_{t}$, using Itô's lemma we get that the density process of $Q_{\lambda} \triangleq \lambda Q+(1-\lambda) P$ is $\mathcal{E}\left(q_{\lambda} \cdot B\right)$ with

$$
q_{\lambda}(t)=\frac{\lambda L_{t} q_{t}}{\lambda L_{t}+(1-\lambda)} 1_{\{t \leq \tau\}},
$$

where $\tau=\inf \left\{t \in[0, T] \mid L_{t}=0\right\} \wedge T$ is a stopping time.

Then from the convexity of $f$ :

$$
\begin{aligned}
C_{0}\left(Q_{\lambda}\right) & =E_{Q_{\lambda}}\left[\int_{0}^{T} f\left(q_{\lambda}(u)\right) d u\right] \\
& \leq E_{Q_{\lambda}}\left[\int_{0}^{\tau} \frac{\lambda L_{t}}{\lambda L_{t}+(1-\lambda)} f(q(t)) d t\right] \\
& =E_{P}\left[\int_{0}^{\tau} \lambda L_{t} f(q(t)) d t\right] \\
& =E_{Q}\left[\int_{0}^{\tau} \lambda f(q(t)) d t\right] \\
& =\lambda C_{0}(Q),
\end{aligned}
$$

where $\lambda \in[0,1]$, we deduce that $\varlimsup_{\lambda \rightarrow 1} C_{0}\left(Q_{\lambda}\right) \leq C_{0}(Q)$.

Notice that for any $\lambda \in[0,1), Q_{\lambda}$ is equivalent to $P$. Thus

$$
\inf \left\{E_{Q}\left[\xi+\int_{0}^{T} f\left(q_{u}\right) d u\right] \mid Q \in \mathcal{P}\right\} \geq \inf \left\{E_{Q}\left[\xi+\int_{0}^{T} f\left(q_{u}\right) d u\right] \mid Q \sim P\right\} .
$$

Since $\{Q \mid Q \sim P\} \subseteq\{Q \mid Q \ll P\}$, we have

$$
U_{0}(\xi)=\inf \left\{E_{Q}\left[\xi+\int_{0}^{T} f\left(q_{u}\right) d u\right] \mid Q \in \mathcal{P}\right\} .
$$

Remark 2.1. The function $C_{0}: \mathcal{P} \rightarrow \overline{R_{+}}$is lower semi-continuous (just use Fatou's lemma) and convex. A duality argument then shows that for $Q \in \mathcal{P}$

$$
C_{0}(Q)=\sup \left\{E_{Q}[-\xi] \mid U_{0}(\xi) \geq 0\right\}
$$

In other words $C_{0}$ is the minimal penalty function as defined in Föllmer-Schied [9]. We also remark that for $Q \ll P$, the previous reasoning and the lower semi-continuity imply $C_{0}\left(Q_{\lambda}\right) \rightarrow C_{0}(Q)$.

However, for a stopping time $\sigma, U_{\sigma}(\xi)$ cannot be the essential infimum over $\mathcal{P} P$ a.s. Instead, by the similar technique as that in Lemma 2.1, we have: 
Remark 2.2. For any measure $Q^{*} \in \mathcal{P}$ and $\xi \in L^{\infty}\left(\mathcal{F}_{T}\right)$,

$$
U_{\sigma}(\xi)=\operatorname{ess.inf}\left\{E_{Q}\left[\xi+\int_{\sigma}^{T} f\left(q_{u}\right) d u \mid \mathcal{F}_{\sigma}\right] \mid Q \in \mathcal{P}, Q \sim P \text { on } \mathcal{F}_{\sigma}\right\}, \quad P \text { a.s. }
$$

for any stopping time $\sigma \in[0, T]$ and,

$$
U_{\sigma}(\xi)=\operatorname{ess.inf}\left\{E_{Q}\left[\xi+\int_{\sigma}^{T} f\left(q_{u}\right) d u \mid \mathcal{F}_{\sigma}\right] \mid Q \in \mathcal{P}, Q^{*} \ll Q\right\}, \quad Q^{*} \text { a.s. }
$$

Proposition 2.1. For any $\xi \in L^{\infty}\left(\mathcal{F}_{T}\right)$, the dynamic utility function $U$ defined by (2.1) has the following properties:

1) For all $Q \ll P$, we have that $U_{t}(\xi)+\int_{0}^{\tau \wedge t} f\left(q_{u}\right) d u$ is a $Q$-submartingale where $\tau=\inf \left\{t \in[0, T] \mid L_{t}=0\right\}$.

2) If there is a probability measure $Q \ll P$ with $U_{0}(\xi)=E_{Q}\left[\xi+\int_{0}^{\tau} f\left(q_{u}\right) d u\right]$, then $U_{t}(\xi)+\int_{0}^{\tau \wedge t} f\left(q_{u}\right) d u$ is a $Q$-martingale.

Proof. 1) For any $s<t$, it follows from Remark 2.2 that for any $Q \ll P$,

$$
\begin{aligned}
& E_{Q}\left[U_{t}(\xi)+\int_{\tau \wedge s}^{\tau \wedge t} f\left(q_{u}\right) d u \mid \mathcal{F}_{s}\right] \\
= & E_{Q}\left[\left(\operatorname{ess.inf}_{Q^{\prime} \sim P}\left\{E_{Q^{\prime}}\left[\xi+\int_{t}^{T} f\left(q_{u}^{\prime}\right) d u \mid \mathcal{F}_{t}\right]\right\}+\int_{\tau \wedge s}^{\tau \wedge t} f\left(q_{u}\right) d u\right) \mid \mathcal{F}_{s}\right] \\
\geq & \text { ess.inf }\left\{E_{Q^{\prime \prime}}\left[\xi+\int_{\tau \wedge s}^{T} f\left(q_{u}^{\prime \prime}\right) d u \mid \mathcal{F}_{s}\right] \mid q_{u}^{\prime \prime}=q_{u}^{\prime}+1_{\{\tau \wedge s \leq u \leq t\}}\left(q_{u}-q_{u}^{\prime}\right)\right\} \\
\geq & \operatorname{ess.inf}\left\{E_{Q^{\prime \prime}}\left[\xi+\int_{\tau \wedge s}^{T} f\left(q_{u}^{\prime \prime}\right) d u \mid \mathcal{F}_{s}\right] \mid Q^{\prime \prime} \in \mathcal{P}, Q \ll Q^{\prime \prime}\right\} \\
\geq & U_{s}(\xi), Q \text { a.s. }
\end{aligned}
$$

Hence,

$$
U_{s}(\xi)+\int_{0}^{\tau \wedge s} f\left(q_{u}\right) d u \leq E_{Q}\left[U_{t}(\xi)+\int_{0}^{\tau \wedge t} f\left(q_{u}\right) d u \mid \mathcal{F}_{s}\right], Q \text { a.s. }
$$

Therefore, we have $U_{t}(\xi)+\int_{0}^{\tau \wedge t} f\left(q_{u}\right) d u$ is a $Q$-submartingale.

2) As $Q$ is absolutely continuous with respect to $P$, it follows from the result we just proved, that

$$
U_{0}(\xi) \leq E_{Q}\left[U_{t}(\xi)+\int_{0}^{\tau \wedge t} f\left(q_{u}\right) d u\right] .
$$

Combining $U_{0}(\xi)=E_{Q}\left[\xi+\int_{0}^{\tau} f\left(q_{u}\right) d u\right]$ with the inequality (2.5), we have

$$
E_{Q}\left[\xi+\int_{\tau \wedge t}^{\tau} f\left(q_{u}\right) d u\right] \leq E_{Q}\left[U_{t}(\xi)\right]
$$

This implies that

$$
U_{t}(\xi)=E_{Q}\left[\xi+\int_{\tau \wedge t}^{\tau} f\left(q_{u}\right) d u \mid \mathcal{F}_{t}\right], Q \text { a.s. }
$$

Thus $U_{t}(\xi)+\int_{0}^{\tau \wedge t} f\left(q_{u}\right) d u=E_{Q}\left[\xi+\int_{0}^{\tau} f\left(q_{u}\right) d u \mid \mathcal{F}_{t}\right]$ is a $Q$ - martingale. 
Remark 2.3. In the above proposition, $\tau$ can be replaced by $T$ since $Q[\tau=T]=1$.

Remark 2.4. In particular, we have that the process $\left\{U_{t}(\xi), t \in[0, T]\right\}$ is a $P$ submartingale. Thus there exists a càdlàg version.

For any $\xi \in L^{\infty}\left(\mathcal{F}_{T}\right),\left|U_{t}(\xi)\right| \leq\|\xi\|_{\infty}$. So applying the Doob-Meyer decomposition theorem, there exists a unique nondecreasing predictable process $\left\{A_{t}\right\}_{0 \leq t \leq T}$ with $A_{0}=$ 0 and a continuous martingale $\left\{M_{t}\right\}_{0 \leq t \leq T}$ with $M_{0}=0$, such that

$$
U_{t}(\xi)=U_{0}(\xi)+A_{t}-M_{t}
$$

Lemma 2.2. For all $\xi \in L^{\infty}\left(\mathcal{F}_{T}\right)$, the martingale part $\left\{M_{t}\right\}_{0 \leq t \leq T}$ of $U(\xi)$ induced by the Doob-Meyer decomposition theorem is a BMO-martingale.

Proof. For a given $\xi \in L^{\infty}\left(\mathcal{F}_{T}\right),\left|U_{t}(\xi)\right| \leq\|\xi\|_{\infty}$. Then applying Itô's formula to $\left(U_{t}(\xi)+\|\xi\|_{\infty}\right)^{2}$, we get

$$
\begin{aligned}
& \left(U_{t}(\xi)+\|\xi\|_{\infty}\right)^{2}+\int_{t}^{T} d\langle M, M\rangle_{s} \\
= & \left(\xi+\|\xi\|_{\infty}\right)^{2}-2 \int_{t}^{T}\left(U_{s-}(\xi)+\|\xi\|_{\infty}\right) d A_{s}-\int_{t}^{T} d K_{s} \\
& +2 \int_{t}^{T}\left(U_{s-}(\xi)+\|\xi\|_{\infty}\right) d M_{s},
\end{aligned}
$$

where

$$
\begin{aligned}
K_{s}:= & \sum_{r \leq s}\left\{\left(U_{r}(\xi)+\|\xi\|_{\infty}\right)^{2}-\left(U_{r-}(\xi)+\|\xi\|_{\infty}\right)^{2}\right. \\
& \left.-2\left(U_{r-}(\xi)+\|\xi\|_{\infty}\right)\left(U_{r}(\xi)-U_{r-}(\xi)\right)\right\} \\
= & \sum_{r \leq s}\left(U_{r}(\xi)-U_{r-}(\xi)\right)^{2}
\end{aligned}
$$

is an increasing process. Hence,

$$
\left(U_{t}(\xi)+\|\xi\|_{\infty}\right)^{2}+\int_{t}^{T} d\langle M, M\rangle_{s} \leq\left(\xi+\|\xi\|_{\infty}\right)^{2}+2 \int_{t}^{T}\left(U_{s-}(\xi)+\|\xi\|_{\infty}\right) d M_{s}
$$

from which we deduce, for any stopping time $0 \leq \sigma \leq T$,

$$
E\left[\int_{\sigma}^{T} d\langle M, M\rangle_{t} \mid \mathcal{F}_{\sigma}\right] \leq 4\|\xi\|_{\infty}^{2}
$$

Therefore, $\|M\|_{B M O_{2}} \leq 2\|\xi\|_{\infty}$ which completes the proof. 
The predictable representation theorem implies that there exists a predictable process $Z \in \mathcal{L}_{\mathcal{F}}^{2}\left(0, T ; \mathbb{R}^{d}\right)$ such that

$$
M_{t}=\int_{0}^{t} Z_{s} d B_{s}
$$

So we get

$$
U_{t}(\xi)=U_{0}(\xi)+A_{t}-\int_{0}^{t} Z_{s} d B_{s}
$$

If $g: R^{d} \rightarrow R_{+} \cup\{\infty\}$ is the Fenchel-Legendre transform of $f$ :

$$
g(z)=\sup _{x \in R^{d}}(z x-f(x)),
$$

then $g$ is also convex and $g(0)=0$.

We make the standard assumption such that both $f$ and $g$ are finite. We do not treat the case where $f$ or $g$ can take the value $+\infty$. This case is similar and only requires cosmetic changes. To make the paper simpler, we dropped this more general case.

Theorem 2.1. Let $U$ be the dynamic utility function defined by (2.1) and let $U_{0}(\xi)+$ $A_{t}-\int_{0}^{t} Z_{u} d B_{u}$ be its decomposition.

1) We have

$$
d A_{t} \geq g\left(Z_{t}\right) d t, P \text { a.s. }
$$

2) Suppose that for some $\xi \in L^{\infty}\left(\mathcal{F}_{T}\right)$ there is a probability measure $Q^{*} \sim P$ with $U_{0}(\xi)=E_{Q^{*}}\left[\xi+\int_{0}^{T} f\left(q_{u}^{*}\right) d u\right]$, then $d A_{t}=g\left(Z_{t}\right) d t$ and

$$
U_{t}(\xi)=U_{0}(\xi)+\int_{0}^{t} g\left(Z_{u}\right) d u-\int_{0}^{t} Z_{u} d B_{u} .
$$

Proof. 1) For $\xi \in L^{\infty}\left(\mathcal{F}_{T}\right)$ and any $Q \sim P$, it follows from the decomposition that

$$
\begin{aligned}
d U_{t}(\xi)+f\left(q_{t}\right) d t & =d A_{t}-Z_{t} d B_{t}+f\left(q_{t}\right) d t \\
& =d A_{t}-Z_{t} q_{t} d t+f\left(q_{t}\right) d t-Z_{t} d B_{t}^{Q}
\end{aligned}
$$

where $B^{Q}$ is a $Q-$ Brownian motion. This implies that $d A_{t}-Z_{t} q_{t} d t+f\left(q_{t}\right) d t$ defines a non-negative measure since $U_{t}(\xi)+\int_{0}^{\tau \wedge t} f\left(q_{u}\right) d u$ is a $Q$-submartingale for any $Q \sim P$. Hence

$$
d A_{t} \geq Z_{t} q_{t} d t-f\left(q_{t}\right) d t
$$


By taking $q^{n}=g^{\prime}(Z) 1_{\{|Z| \leq n\}}$ in the above inequality and by letting $n$ tend to infinity, we get $d A_{t} \geq g\left(Z_{t}\right) d t$.

2) If for $\xi$, there is a measure $Q^{*} \sim P$ with $U_{0}(\xi)=E_{Q^{*}}\left[\xi+\int_{0}^{T} f\left(q_{u}^{*}\right) d u\right]$, then it follows from Proposition 2.1 that $U_{t}(\xi)+\int_{0}^{t} f\left(q_{u}^{*}\right) d u$ is a $Q^{*}$-martingale. Thus applying (2.13) with $Q^{*}$, we get

$$
d A_{t}=\left(Z_{t} q_{t}^{*}-f\left(q_{t}^{*}\right)\right) d t \quad Q^{*} \text { a.s. }
$$

Since $Q^{*} \sim P$, we have

$$
d A_{t}=\left(Z_{t} q_{t}^{*}-f\left(q_{t}^{*}\right)\right) d t \quad P \text { a.s. }
$$

Finally combining (2.10) and (2.14) with the definition of $g$, it follows that

$$
g\left(Z_{t}\right) d t \geq\left(Z_{t} q_{t}^{*}-f\left(q_{t}^{*}\right)\right) d t=d A_{t} \geq g\left(Z_{t}\right) d t \quad P \text { a.s. }
$$

In general we can decompose $A$ further and get:

Corollary 2.1. For any $\xi \in L^{\infty}\left(\mathcal{F}_{T}\right)$, there exists an increasing predictable process $\left\{C_{t}\right\}_{0 \leq t \leq T}$ with $C_{0}=0$ such that

$$
U_{t}(\xi)=U_{0}(\xi)+\int_{0}^{t} g\left(Z_{u}\right) d u-\int_{0}^{t} Z_{u} d B_{u}+C_{t}
$$

Our main result is the following.

Theorem 2.2. Let $U$ be the dynamic utility function defined by (2.1). Then the following are equivalent:

1. $\varliminf_{|x| \rightarrow \infty} \frac{f(x)}{|x|^{2}}>0$;

2. $\varlimsup_{|z| \rightarrow \infty} \frac{g(z)}{|z|^{2}}<\infty$;

3. For all $k>0$, the set $\left\{Q \mid C_{0}(Q) \leq k\right\}$ is weakly compact;

4. For all $\xi \in L^{\infty}\left(\mathcal{F}_{T}\right)$, there exists a measure $Q \ll P$ such that $U_{0}(\xi)=E_{Q}[\xi+$ $\left.\int_{0}^{T} f\left(q_{u}\right) d u\right]$

5. For all $\xi \in L^{\infty}\left(\mathcal{F}_{T}\right)$, there exists a measure $Q \sim P$ such that $U_{0}(\xi)=E_{Q}[\xi+$ $\left.\int_{0}^{T} f\left(q_{u}\right) d u\right]$

6. For all $\xi \in L^{\infty}\left(\mathcal{F}_{T}\right)$, the BSDE $d Y_{t}=g\left(Z_{t}\right) d t-Z_{t} d B_{t}$ has a unique bounded solution with $Y_{T}=\xi$. 


\section{7. $U_{0}$ is strictly monotone.}

Proof. $1 \Leftrightarrow 2$ : Point 1 implies that there exist positive constants $a, b \in R_{+}$such that $f(x) \geq a|x|^{2}-b$. We then get

$$
g(z)=\sup _{x \in R^{d}}(z x-f(x)) \leq \sup _{x \in R^{d}}\left(z x-a|x|^{2}+b\right) \leq \frac{1}{4 a}|z|^{2}+b
$$

which shows that $\varlimsup_{z \rightarrow \infty} \frac{g(z)}{|z|^{2}}<\infty$. The proof of the implication $2 \Rightarrow 1$ is similar.

$1 \Rightarrow 3$ : It suffices to verify that for any $k>0,\left\{\frac{d Q}{d P} \mid C_{0}(Q)=E_{Q}\left[\int_{0}^{T} f\left(q_{u}\right) d u\right] \leq k\right\}$ is uniformly integrable. The Dunford-Pettis theorem then shows that the set is weakly compact.

Since $f(x) \geq a|x|^{2}-b$, we get

$$
k \geq E_{Q}\left[\int_{0}^{T} f\left(q_{u}\right) d u\right] \geq a E_{Q}\left[\int_{0}^{T}\left|q_{u}\right|^{2} d u\right]-b .
$$

Therefore,

$$
\frac{1}{2} E_{Q}\left[\int_{0}^{T}\left|q_{u}\right|^{2} d u\right] \leq \alpha
$$

where $\alpha=\frac{k+b}{2 a}$ is a positive constant independent of $Q$. It follows from

$$
\begin{aligned}
\frac{1}{2} E_{Q}\left[\int_{0}^{T}\left|q_{u}\right|^{2} d u\right] & =E_{Q}\left[\int_{0}^{T} q_{u} d B_{u}^{Q}+\frac{1}{2} \int_{0}^{T}\left|q_{u}\right|^{2} d u\right] \\
& =E_{Q}\left[\int_{0}^{T} q_{u} d B_{u}-\frac{1}{2} \int_{0}^{T}\left|q_{u}\right|^{2} d u\right] \\
& =E_{Q}\left[\log \frac{d Q}{d P}\right]
\end{aligned}
$$

that for any $k>0$,

$$
\left\{\frac{d Q}{d P} \mid E_{Q}\left[\int_{0}^{T} f\left(q_{u}\right) d u\right] \leq k\right\} \subseteq\left\{\frac{d Q}{d P} \mid E_{P}\left[\frac{d Q}{d P} \log \frac{d Q}{d P}\right] \leq \alpha\right\} .
$$

From the de la Vallée Poussin theorem, we conclude that

$$
\left\{\frac{d Q}{d P} \mid E_{Q}\left[\int_{0}^{T} f\left(q_{u}\right) d u\right] \leq k\right\} \text { is uniformly integrable. }
$$

$3 \Rightarrow 1$ We prove it by the contradiction. Suppose $\underline{\lim }_{|x| \rightarrow \infty} \frac{f(x)}{|x|^{2}}=0$, then there exists a sequence $\left\{x_{n}\right\}_{n=0}^{\infty}$ such that $\lim _{n \rightarrow \infty}\left|x_{n}\right|=\infty$ and $\lim _{n \rightarrow \infty} \frac{f\left(x_{n}\right)}{\left|x_{n}\right|^{2}}=0$. Put $q_{n}=x_{n} 1_{\left[0, \delta_{n} \wedge T\right]}$ where $\delta_{n}=1 /\left(\sqrt{\frac{f\left(x_{n}\right)}{\left|x_{n}\right|^{2}}}\left|x_{n}\right|^{2}\right)$. It follows from

$$
C_{0}\left(Q_{n}\right)=E_{Q_{n}}\left[\int_{0}^{T} f\left(q_{n}(u)\right) d u\right] \leq \sqrt{\frac{f\left(x_{n}\right)}{\left|x_{n}\right|^{2}}} \rightarrow 0,
$$


that for all $k>0$, there exists $N>0$ such that the sequence $\left\{\frac{d Q_{n}}{d P}\right\}_{n=N}^{\infty} \subseteq\left\{\frac{d Q}{d P} \mid C_{0}(Q) \leq\right.$ $k\}$. Furthermore, we have

$$
\int_{0}^{T}\left|q_{n}\right|^{2}(u) d u=\left(1 / \sqrt{\frac{f\left(x_{n}\right)}{\left|x_{n}\right|^{2}}}\right) \wedge\left(x_{n}^{2} T\right) \rightarrow \infty,
$$

which shows that $\frac{d Q_{n}}{d P}=\mathcal{E}\left(q_{n} \cdot B\right)_{T} \rightarrow 0$, a.s. as $n \rightarrow \infty$. Thus $\left\{\frac{d Q_{n}}{d P}\right\}_{n=N}^{\infty}$ is not uniformly integrable.

$3 \Leftrightarrow 4$ : It is a conclusion induced by the James' theorem as shown in JouiniSchachermayer-Touzi's work [11].

$4 \Leftrightarrow 5$ : It is obvious that point 5 implies point 4 . For the proof of the inverse implication, we use the fact that condition 4 is equivalent to condition 2 . In this case, by convexity, there exists a positive constant $c$ such that $\left|g^{\prime}(z)\right| \leq c(|z|+1)$. For any $\xi \in L^{\infty}\left(\mathcal{F}_{T}\right)$, there is a measure $Q \ll P$ such that $U_{0}(\xi)=E_{Q}\left[\xi+\int_{0}^{T} f\left(q_{u}\right) d u\right]$, then, by Proposition 2.1, $U_{t}(\xi)+\int_{0}^{\tau \wedge t} f\left(q_{u}\right) d u$ is a $Q$-martingale where $\tau=\inf \{t \in$ $\left.[0, T] \mid \mathcal{E}(q \cdot B)_{t}=0\right\} \wedge T$. It follows from (2.13) that

$$
d A_{t}=\left(Z_{t} q_{t}-f\left(q_{t}\right)\right) d t \quad m \otimes Q \text { a.s. on }[0, \tau]
$$

where $m$ is the Lebesgue measure on $[0, T]$. Since $d A_{t} \geq g\left(Z_{t}\right) d t, m \otimes Q$ a.s., we get

$$
g\left(Z_{t}\right)=Z_{t} q_{t}-f\left(q_{t}\right) \quad m \otimes Q \text { a.s. }
$$

which implies $q_{t}=g^{\prime}\left(Z_{t}\right)$ on $[0, \tau]$. We then have

$$
\begin{aligned}
\int_{0}^{\tau}\left|q_{u}\right|^{2} d u & =\int_{0}^{\tau}\left(g^{\prime}\left(Z_{u}\right)\right)^{2} d u \\
& \leq c^{2} \int_{0}^{\tau}\left(1+\left|Z_{u}\right|\right)^{2} d u<\infty
\end{aligned}
$$

which means $P\left\{\frac{d Q}{d P}=0\right\}=P\left\{\int_{0}^{\tau}\left|q_{u}\right|^{2} d u=\infty\right\}=0$. Hence $Q \sim P$.

$5 \Rightarrow 6$ : For a given $\xi \in L^{\infty}\left(\mathcal{F}_{T}\right)$, if there exists a measure $Q \sim P$ such that $U_{0}(\xi)=$ $E_{Q}\left[\xi+\int_{0}^{T} f\left(q_{u}\right) d u\right]$, it follows from Lemma 2.1 that $\left\{U_{t}, Z_{t}\right\}_{0 \leq t \leq T}$ is a solution of the following BSDE:

$$
\left\{\begin{array}{l}
d Y_{t}=g\left(z_{t}\right) d t-z_{t} d B_{t}, \quad 0 \leq t \leq T \\
Y_{T}=\xi, \quad \xi \in L^{\infty}\left(\mathcal{F}_{T}\right) \\
Y \text { is bounded }
\end{array}\right.
$$


where $E\left[\int_{0}^{T}\left|z_{t}\right|^{2} d t\right]<\infty$ and $E\left[\int_{0}^{T} g\left(z_{t}\right) d t\right]<\infty$. Since, as we have proved above, condition 5 implies $\varlimsup_{z \rightarrow \infty} \frac{g(z)}{|z|^{2}}<\infty$, the BSDE has a unique bounded solution according to Kobylanski [12].

$6 \Rightarrow 2$ We will prove this in the next section. See Theorem 3.1 .

$5 \Rightarrow 7$ For any $\xi \in L^{\infty}\left(\mathcal{F}_{T}\right)$, there exists an equivalent measure $Q \sim P$ such that $U_{0}(\xi)=E_{Q}\left[\xi+\int_{0}^{T} f\left(q_{u}\right) d u\right]$ with $\frac{d Q}{d P}=\mathcal{E}(q \cdot B)$.

Suppose that $U_{0}(\eta)=U_{0}(\xi)$ for some $\eta \in L^{\infty}\left(\mathcal{F}_{T}\right)$ with $\eta \leq \xi, P$ a.s. Since

$$
U_{0}(\eta) \leq E_{Q}\left[\eta+\int_{0}^{T} f\left(q_{u}\right) d u\right] \leq E_{Q}\left[\xi+\int_{0}^{T} f\left(q_{u}\right) d u\right]=U_{0}(\xi)
$$

we have $E_{Q}[\xi-\eta]=0$, hence $\xi=\eta, Q$ a.s. Thus $\xi=\eta, P$ a.s. and $U_{0}$ is strictly monotone.

\section{$7 \Rightarrow 2$ See Remark 3.2 , Remark 3.5 or Example 3.1 .}

We have proved that in the case when the generator $g$ is at most quadratic, the dynamic utility function $U$ is the solution of BSDE (2.19). In general, however, we have the following inequality.

Lemma 2.3. For any $\xi \in L^{\infty}\left(\mathcal{F}_{T}\right)$, if $B S D E$ (2.19) has a bounded solution $Y$, then we have $U(\xi) \geq Y$.

Proof. $Y$ is bounded. The following calculation is therefore justified:

$$
\begin{aligned}
E_{Q}\left[\xi+\int_{t}^{T} f\left(q_{u}\right) d u \mid \mathcal{F}_{t}\right] & =Y_{t}+E_{Q}\left[\int_{t}^{T} g\left(Z_{u}\right) d u-\int_{t}^{T} Z_{u} d B_{u}+\int_{t}^{T} f\left(q_{u}\right) d u \mid \mathcal{F}_{t}\right] \\
& =Y_{t}+E_{Q}\left[\int_{t}^{T}\left[g\left(Z_{u}\right)-Z_{u} q_{u}+f\left(q_{u}\right)\right] d u \mid \mathcal{F}_{t}\right] \\
& \geq Y_{t}, \text { for any } Q \sim P \text { with } E_{Q}\left[\int_{0}^{T} f\left(q_{u}\right) d u\right]<\infty .
\end{aligned}
$$

\section{Backward SDEs with superquadratic growth.}

In this section, we discuss the following $\operatorname{BSDE}(g, \xi)$ :

$$
\left\{\begin{array}{l}
d Y_{t}=g\left(Z_{t}\right) d t-Z_{t} d B_{t} \\
Y_{T}=\xi, \quad \xi \in L^{\infty}\left(\mathcal{F}_{T}\right)
\end{array}\right.
$$


where $g: R^{d} \rightarrow R_{+} \cup\{+\infty\}$ is convex with $g(0)=0$ and superquadratic $\varlimsup_{|z| \rightarrow \infty} \frac{g(z)}{|z|^{2}}=$ $\infty$. A pair of predictable processes $(Y, Z)$ is called a bounded solution to BSDE (3.1) if

$Y: \Omega \times[0, T] \rightarrow R$ is bounded and

$Z: \Omega \times[0, T] \rightarrow R^{d}$ is such that $E\left[\int_{0}^{T} g\left(Z_{t}\right) d t\right]<\infty$.

Here for simplicity, we consider the BSDE with $d=1$. However, the results remain valid for $d>1$.

\subsection{Non-existence of the solution}

Different from the BSDEs with at most quadratic growth, the solution to the BSDE with super-quadratic growth does not always exist.

Theorem 3.1. (Non-existence) There exists $\eta \in L^{\infty}\left(\mathcal{F}_{T}\right)$ such that BSDE(3.1) with superquadratic growth has no bounded solution.

Proof. The proof is divided into 4 steps.

Step 1. We construct a pair of processes $(X, Z)$, a measure $Q$ as well as a bounded random variable $\xi$.

Since $\varlimsup_{|z| \rightarrow \infty} \frac{g(z)}{|z|^{2}}=\infty$, there exists a sequence $\left\{z_{k}\right\}_{k=1}^{\infty}$ such that $\lim _{k \rightarrow \infty}\left|z_{k}\right|=\infty$ and $g\left(z_{k}\right) \geq k\left|z_{k}\right|^{2}$. Without loss of generality, we suppose $z_{k}>0$. The other case is left to the reader. Thus we have

$$
g^{\prime}\left(z_{k}\right) \geq \frac{g\left(z_{k}\right)}{z_{k}} \geq k \cdot z_{k}
$$

We put $Z_{u} \triangleq \sum_{n=1}^{\infty} z_{n} 1_{\left[\sum_{k<n} \delta_{k}, \sum_{k \leq n} \delta_{k}\right)}(u)$ where

$$
\delta_{k}=\frac{1}{\alpha z_{k} g^{\prime}\left(z_{k}\right) k^{2}}
$$

and we set

$$
\alpha=\sum_{k=1}^{\infty} \frac{1}{T z_{k} g^{\prime}\left(z_{k}\right) k^{2}}<\infty
$$

in order to have

$$
\sum_{k \geq 1} \delta_{k}=\sum_{k \geq 1} \frac{1}{\alpha z_{k} g^{\prime}\left(z_{k}\right) k^{2}}=T
$$

Then from (3.2), we have

$$
\int_{0}^{T} g\left(Z_{u}\right) d u=\sum_{k \geq 1} g\left(z_{k}\right) \delta_{k} \leq \sum_{k \geq 1} z_{k} g^{\prime}\left(z_{k}\right) \delta_{k}=\sum_{k \geq 1} \frac{1}{\alpha k^{2}}<\infty,
$$




$$
\int_{0}^{T}\left|Z_{u}\right|^{2} d u=\sum_{k \geq 1}\left|z_{k}\right|^{2} \delta_{k} \leq \sum_{k \geq 1} z_{k} g^{\prime}\left(z_{k}\right) \delta_{k} \frac{1}{k}=\sum_{k \geq 1} \frac{1}{\alpha k^{3}}<\infty .
$$

Let $q_{t}=g^{\prime}\left(Z_{t}\right)$. It follows from

$$
\int_{0}^{T}\left|q_{u}\right|^{2} d u=\sum_{k \geq 1}\left(g^{\prime}\left(z_{k}\right)\right)^{2} \delta_{k} \geq \sum_{k \geq 1} k g^{\prime}\left(z_{k}\right) z_{k} \delta_{k}=\sum_{k \geq 1} \frac{1}{\alpha k}=+\infty,
$$

that $\lim _{t \rightarrow T} \mathcal{E}(q \cdot B)_{t}=0$ and $\mathcal{E}(q \cdot B)_{t}>0, P$ a.s. for any $t<T$.

Let $X_{t}=\int_{0}^{t} g\left(Z_{u}\right) d u-\int_{0}^{t} Z_{u} d B_{u}$. We stop $X$ at a random time $\sigma$

$$
\sigma \triangleq \inf \left\{t \in[0, T] \mid \mathcal{E}(q \cdot B)_{t} \geq n\right\} \wedge \inf \left\{t \in[0, T]|| X_{t} \mid \geq n\right\} \wedge T
$$

where $n$ is a positive constant which is sufficiently large to ensure that $P(\sigma=T)>0$. We then set a measure $Q^{*}$ with $E_{P}\left[\frac{d Q^{*}}{d P} \mid \mathcal{F}_{t}\right]=\mathcal{E}\left(q^{*} \cdot B\right)_{t}$ and $q_{t}^{*}=g^{\prime}\left(Z_{t}\right) 1_{\{t \leq \sigma\}}$.

We define $\xi=X_{\sigma} \in L^{\infty}\left(\mathcal{F}_{T}\right)$.

Step 2. The measure $Q^{*} \ll P$ but it is not equivalent to $P$.

Set $A_{1}=\{\sigma=T\}$. Then

$$
Q^{*}\left(A_{1}\right)=\int_{A_{1}} \mathcal{E}(q \cdot B)_{\sigma} d P=\int_{A_{1}} \mathcal{E}(q \cdot B)_{T} d P=0
$$

while $P\left(A_{1}\right)>0$. Thus we have $Q^{*} \nsim P$ and $Q^{*} \ll P$. However, $Q^{*} \otimes m \sim P \otimes m$ where $m$ is the Lebesgue measure since $Q^{*} \sim P$ on $\mathcal{F}_{t}$ for all $t<T$. Clearly $\left(X_{t}^{\sigma}, Z_{t} 1_{\{t \leq \sigma\}}\right)_{0 \leq t \leq T}$ is a bounded solution of $\operatorname{BSDE}(g, \xi)$ where $X_{t}^{\sigma}=X_{\sigma \wedge t}$.

Step 3. In this step we prove that the dynamic utility function $U(\xi)$ is the bounded solution of $\operatorname{BSDE}(g, \xi)$ (3.1) and $U_{t}(\xi)=E_{Q^{*}}\left[\xi+\int_{t}^{T} f\left(q_{u}^{*}\right) d u \mid \mathcal{F}_{t}\right]$ for any $t<T$.

As $X^{\sigma}$ is a bounded solution of $\operatorname{BSDE}(g, \xi)$, we get

$$
\begin{aligned}
U_{t}(\xi) & \leq E_{Q^{*}}\left[\xi+\int_{t}^{T} f\left(q_{u}^{*}\right) d u \mid \mathcal{F}_{t}\right] \\
& =E_{Q^{*}}\left[\xi+\int_{t \wedge \sigma}^{\sigma} f\left(q_{u}^{*}\right) d u \mid \mathcal{F}_{t}\right] \\
& =X_{t}^{\sigma}+E_{Q^{*}}\left[\int_{t \wedge \sigma}^{\sigma}\left(f\left(q_{u}^{*}\right)+g\left(Z_{u}\right)\right) d u-\int_{t \wedge \sigma}^{\sigma} Z_{u} d B_{u} \mid \mathcal{F}_{t}\right] \\
& =X_{t}^{\sigma}+E_{Q^{*}}\left[\int_{t \wedge \sigma}^{\sigma}\left[f\left(q_{u}^{*}\right)+g\left(Z_{u}\right)-Z_{u} q_{u}^{*}\right] d u \mid \mathcal{F}_{t}\right] \\
& =X_{t}^{\sigma}, \quad Q^{*} \text { a.s. }
\end{aligned}
$$

hence $P$ a.s. because $Q^{*} \sim P$ on $\mathcal{F}_{t}$ for $t<T$. Combining Lemma 2.3 with inequality (3.4), we deduce that

$$
U_{t}(\xi)=E_{Q^{*}}\left[\xi+\int_{t}^{T} f\left(q_{u}^{*}\right) d u \mid \mathcal{F}_{t}\right]=X_{t}^{\sigma}, \quad P \text { a.s. for all } t \in[0, T) .
$$


Set $\eta=\xi+h$ where $h \in L_{+}^{\infty}\left(\mathcal{F}_{T}\right), P[h>0]>0$ and $h \cdot \mathcal{E}\left(q^{*} \cdot B\right)_{\sigma}=0$.

Step 4. We show that $U_{t}(\xi)=U_{t}(\eta), P$ a.s. for any $t<T$ and hence $\operatorname{BSDE}(g, \eta)$ has no solution.

It follows from $\eta=\xi, Q^{*}$-a.s. that

$$
\begin{aligned}
U_{t}(\eta) & \leq E_{Q^{*}}\left[\eta+\int_{t}^{T} f\left(q_{u}^{*}\right) d u \mid \mathcal{F}_{t}\right] \\
& =E_{Q^{*}}\left[\xi+\int_{t}^{T} f\left(q_{u}^{*}\right) d u \mid \mathcal{F}_{t}\right] \\
& =U_{t}(\xi) \quad \text { for any } t<T .
\end{aligned}
$$

Notice that $U$ is monotone, i.e., $U_{t}(\xi) \leq U_{t}(\eta)$, and so we have $U_{t}(\xi)=U_{t}(\eta), P$ a.s. for any $t<T$.

Suppose $Y$ is a bounded solution of $\operatorname{BSDE}(g, \eta)$, then we have for $t<T$,

$$
X_{t}^{\sigma}=U_{t}(\xi)=U_{t}(\eta) \geq Y_{t}
$$

and hence

$$
\eta=Y_{T}=\lim _{t \rightarrow T} Y_{t} \leq \lim _{t \rightarrow T} X_{t}^{\sigma}=X_{T}^{\sigma}=\xi, P \text { a.s. },
$$

a contradiction to the fact that $P[\eta>\xi]>0$. Therefore, BSDE $(g, \eta)$ has no solution.

Remark 3.1. From this theorem, together with what we have proved in Theorem 2.2 we get that $\operatorname{BSDE}(g, \xi)$ has a solution for all $\xi \in L^{\infty}\left(\mathcal{F}_{T}\right)$ if and only if $g$ is at most quadratic.

Remark 3.2. From the proof, we get $\eta \geq \xi$ with $P(\eta>\xi)>0$ and $U_{0}(\xi)=U_{0}(\eta)$. Thus the utility function $U_{0}$ is NOT strictly monotone when $\underline{\lim }_{|x| \rightarrow \infty} \frac{f(x)}{|x|^{2}}=0$.

Although the BSDE $(g, \xi)$ (3.1) does not always have a solution, in the following case it has.

Definition 3.1. We say that a random variable $\xi \in L^{\infty}\left(\mathcal{F}_{T}\right)$ is minimal if $\eta \leq \xi$ and $P[\eta<\xi]>0$ imply $U_{0}(\eta)<U_{0}(\xi)$.

Theorem 3.2. Let $\xi \in L^{\infty}\left(\mathcal{F}_{T}\right)$ be minimal. Then $U(\xi)$ is a solution of $B S D E(g, \xi)$.

Proof. We prove it by contradiction. Let $\xi \in L^{\infty}\left(\mathcal{F}_{T}\right)$ be minimal and suppose $U(\xi)$ is not a solution of $\operatorname{BSDE}(g, \xi)$. Then it follows from Corollary 2.1 that there 
exists an increasing process $C$ with $C_{0}=0$ such that $P\left[C_{T}>0\right]>0$ and

$$
U_{t}(\xi)=\xi-\int_{t}^{T} g\left(Z_{u}\right) d u+\int_{t}^{T} Z_{u} d B_{u}-C_{T}+C_{t} .
$$

Define $\tau:=\inf \left\{t \in[0, T] \mid C_{t} \geq k\right\} \wedge T$, where $k>0$ is such that $P\left[C_{\tau}>0\right]>0$. Since $C$ may have jumps, $C_{\tau}$ can be unbounded. However, $\tau$ is predictable so there exists $\left\{\tau_{n}\right\}_{n=1}^{\infty}$ such that $\tau_{n} \uparrow \tau$ and $\tau_{n}<\tau$ on $\{\tau>0\}$. It follows that $C_{\tau_{n}} \leq k$ and $P\left[C_{\tau_{n}}>0\right]>0$ for $n$ big enough. Denote by $\sigma$ a stopping time $\tau_{n}$ for $n$ big enough, then we have

$$
U_{t}(\xi)-C_{t}=U_{\sigma}(\xi)-C_{\sigma}-\int_{t}^{\sigma} g\left(Z_{u}\right) d u+\int_{t}^{\sigma} Z_{u} d B_{u}
$$

which implies that $\left(U_{t \wedge \sigma}(\xi)-C_{t \wedge \sigma}, Z_{t} 1_{\{t \leq \sigma\}}\right)_{0 \leq t \leq T}$ is a solution of BSDE $\left(g, U_{\sigma}(\xi)-\right.$ $\left.C_{\sigma}\right)$. Thus by Lemma 2.3 , we deduce

$$
U_{0}(\xi)=U_{0}(\xi)-C_{0} \leq U_{0}\left(U_{\sigma}(\xi)-C_{\sigma}\right)
$$

On the other hand, it is clear that $U_{0}(\xi) \geq U_{0}\left(U_{\sigma}(\xi)-C_{\sigma}\right)$. Therefore, we have

$$
U_{0}(\xi)=U_{0}\left(U_{\sigma}(\xi)-C_{\sigma}\right)
$$

It follows from the above equality, the translability and the time-consistency of the dynamic utility function that

$$
U_{0}(\xi)=U_{0}\left(U_{\sigma}(\xi)-C_{\sigma}\right)=U_{0}\left(U_{\sigma}\left(\xi-C_{\sigma}\right)\right)=U_{0}\left(\xi-C_{\sigma}\right)
$$

This is a contradiction to the fact that $\xi$ is minimal.

Remark 3.3. For $g$ with at most quadratic growth $\overline{\lim }_{|z| \rightarrow \infty} \frac{g(z)}{|z|^{2}}<\infty$, it follows from Theorem 2.2 that $\xi$ is minimal for all $\xi \in L^{\infty}\left(\mathcal{F}_{T}\right)$.

If $g$ is superquadratic, there exists a bounded random variable $\zeta$ such that $U(\zeta)$ is a solution of $\operatorname{BSDE}(g, \zeta)$ and $\zeta$ is not minimal. See Example 3.1 .

\subsection{Non-uniqueness of the Solution}

In this subsection, we shall prove that if the BSDE has a bounded solution, the bounded solution is not unique. The main reason is that the generator $g$ is superquadratic which makes $\int_{0}^{t} g\left(Z_{r}\right) d r$ grow much faster than $\int_{0}^{t} Z_{r} d B_{r}$. Following this observation, we can construct other solutions. 
Theorem 3.3. (Non-uniqueness) If the BSDE $(g, \xi)$ with superquadratic growth has a bounded solution $Y$ for a $\xi \in L^{\infty}\left(\mathcal{F}_{T}\right)$, then for each $y<Y_{0}$, there are infinitely many bounded solutions $\left\{X_{t}\right\}_{0 \leq t \leq T}$ with $X_{0}=y$.

Proof. Suppose $(Y, Z)$ is a bounded solution of $\operatorname{BSDE}(g, \xi)$. Divide the time interval $[0, T]$ into $\left[T\left(1-2^{-n}\right), T\left(1-2^{-n-1}\right)\right)$, where $n=0,1,2, \ldots$ and denote $\alpha_{n}=T\left(1-2^{-n}\right)$. Suppose the new solution $\left(X, Z^{\prime}\right)$ has been constructed on $\left[0, \alpha_{n}\right]$ with $X_{0}=y$ where $y<Y_{0}$ such that $X_{\alpha_{n}} \leq Y_{\alpha_{n}} P$ a.s. Let us construct $\left(X, Z^{\prime}\right)$ on the time interval $\left[\alpha_{n}, \alpha_{n+1}\right)$.

Our idea is the following. Since $g$ is superquadratic, we can construct a process $X_{\alpha_{n}}+V_{t}, t \in\left[\alpha_{n}, \alpha_{n+1}\right)$ such that $\lim _{t \rightarrow \alpha_{n+1}} V_{t}=+\infty, P$ a.s. and for any $0<\varepsilon<1, V_{t}$ exceeds downwards $-2^{-n-1} \varepsilon$ with a very small probability. The fact that the solution $Y$ is bounded implies that it is touched by the process $X_{\alpha_{n}}+V_{t}$ because $X_{\alpha_{n}} \leq Y_{\alpha_{n}}$. We then get a new solution $X_{t}$ on this time interval $\left[\alpha_{n}, \alpha_{n+1}\right]$ by stopping $X_{\alpha_{n}}+V_{t}$ when it reaches $Y$.

First, let us construct the process $V_{t}$.

It follows from $\varlimsup_{z \rightarrow \infty} \frac{g(z)}{|z|^{2}}=\infty$ that there exists a sequence $\left\{x_{k}\right\}_{k=0}^{\infty}$ such that for any $k \geq 0$,

1. $g\left(x_{k}\right) \geq 4^{n} x_{k}^{2}$

2. $x_{k}^{2} \geq \frac{1}{\left(\theta^{k}-\theta^{k+1}\right) \theta^{k} \delta_{n}}$ where $\theta \in(0,1)$ is a constant and $\delta_{n}=\alpha_{n+1}-\alpha_{n}=2^{-n-1} T$.

Set $b_{t}=\sum_{k=0}^{\infty} x_{k} 1_{\left[\alpha_{n+1}-\theta^{k} \delta_{n}, \alpha_{n+1}-\theta^{k+1} \delta_{n}\right)}(t)$ and $V_{t}=\int_{\alpha_{n}}^{t} g\left(b_{u}\right) d u-\int_{\alpha_{n}}^{t} b_{u} d B_{u}$ for any $t \in\left[\alpha_{n}, \alpha_{n+1}\right)$.

We then have for $t \in\left[\alpha_{n+1}-\theta^{N+1} \delta_{n}, \alpha_{n+1}-\theta^{N+2} \delta_{n}\right)$,

$$
\begin{gathered}
\int_{\alpha_{n}}^{t} b_{u}^{2} d u \geq \sum_{k=0}^{N} x_{k}^{2}\left(\theta^{k}-\theta^{k+1}\right) \delta_{n} \geq \sum_{k=0}^{N} \frac{1}{\theta^{k}}, \\
\int_{\alpha_{n}}^{t} g\left(b_{u}\right) d u \geq 4^{n} \int_{\alpha_{n}}^{t} b_{u}^{2} d u \geq 4^{n} \sum_{k=0}^{N} \frac{1}{\theta^{k}} .
\end{gathered}
$$

Thus $\lim _{t \rightarrow \alpha_{n+1}} \int_{\alpha_{n}}^{t} b_{u}^{2} d u=\infty$ and $\lim _{t \rightarrow \alpha_{n+1}} \int_{\alpha_{n}}^{t} g\left(b_{u}\right) d u=\infty$.

Step 1. We have $\lim _{t \rightarrow \alpha_{n+1}} V_{t}=+\infty P$ a.s.

Define $\phi(t)=\int_{\alpha_{n}}^{t} b_{u}^{2} d u$ for $t \in\left[\alpha_{n}, \alpha_{n+1}\right)$. Then $\phi$ is strictly increasing with $\phi\left(\alpha_{n}\right)=0$ and

$$
\lim _{t \rightarrow \alpha_{n+1}} \phi(t)=+\infty
$$


Setting $B_{t}^{*} \triangleq \int_{\alpha_{n}}^{\phi^{-1}(t)} b_{u} d B_{u}$, we get a time changed Brownian motion with respect to the filtration $\left\{\mathcal{F}^{B^{*}}\right\}$. It follows from the construction of $V$ that

$$
V_{t} \geq 4^{n} \phi(t)-B_{\phi(t)}^{*}=\phi(t)\left[4^{n}-\frac{B_{\phi(t)}^{*}}{\phi(t)}\right],
$$

which implies that

$$
\lim _{t \rightarrow \alpha_{n+1}} V_{t}=+\infty, P \text { a.s. }
$$

since

$$
\lim _{t \rightarrow \alpha_{n+1}} \frac{B_{\phi(t)}^{*}}{\phi(t)}=0, P \text { a.s. }
$$

Now we estimate the probability that $V_{t}$ reaches a small negative number $-2^{-n-1} \varepsilon$.

Step 2. Calculate the probability

$$
P\left(\left\{\omega \in \Omega \mid \exists t \in\left[\alpha_{n}, \alpha_{n+1}\right) \text { such that } V_{t}(\omega)<-2^{-n-1} \varepsilon\right\}\right) .
$$

Applying the submartingale inequality, we deduce that

$$
\begin{aligned}
& P\left(\left\{\omega \in \Omega \mid \exists t \in\left[\alpha_{n}, \alpha_{n+1}\right) \text { such that } V_{t}(\omega)<-2^{-n-1} \varepsilon\right\}\right) \\
= & P\left(\left\{\omega \in \Omega \mid \exists t \in\left[\alpha_{n}, \alpha_{n+1}\right) \text { such that } 4^{n} \phi(t)-B_{\phi(t)}^{*}<-2^{-n-1} \varepsilon\right\}\right) \\
= & P\left(\left\{\omega \in \Omega \mid \exists s \in[0, \infty) \text { such that } 4^{n} s-B_{s}^{*}<-2^{-n-1} \varepsilon\right\}\right) \\
\leq & \exp \left\{-2^{n} \varepsilon\right\} .
\end{aligned}
$$

Step 3. Construct the new solution $\left(X_{t}, Z_{t}^{\prime}\right)$ for all $t \in\left[\alpha_{n}, \alpha_{n+1}\right]$.

Define

$$
\tau_{1} \triangleq \inf \left\{t \geq \alpha_{n} \mid V_{t}=-2^{-n-1} \varepsilon\right\} \wedge \alpha_{n+1}
$$

and

$$
\tau_{2} \triangleq \inf \left\{t \geq \alpha_{n} \mid X_{\alpha_{n}}+V_{t} \geq Y_{t}\right\} \wedge \alpha_{n+1}
$$

which are the stopping times when the process $X_{\alpha_{n}}+V_{t}$ touches $X_{\alpha_{n}}-2^{-n-1} \varepsilon$ and $Y_{t}$ respectively. It follows from $\lim _{t \rightarrow \alpha_{n+1}} V_{t}=+\infty P$ a.s. that $P\left[\tau_{2}<\alpha_{n+1}\right]=1$. Define

$$
\tau_{3} \triangleq \inf \left\{t \geq \tau_{1} \mid X_{\alpha_{n}}-2^{-n-1} \varepsilon=Y_{t}\right\} \wedge \alpha_{n+1} .
$$

Now we have three cases

$$
\begin{cases}\tau_{1}<\tau_{2}, \tau_{3}<\alpha_{n+1}, & \text { put } Z_{t}^{\prime}(\omega)=b_{t} 1_{\left\{t \leq \tau_{1}\right\}}+Z_{t} 1_{\left\{t>\tau_{3}\right\}} \\ \tau_{1}<\tau_{2}, \tau_{3}=\alpha_{n+1}, & \text { put } Z_{t}^{\prime}(\omega)=b_{t} 1_{\left\{t \leq \tau_{1}\right\}} ; \\ \tau_{1} \geq \tau_{2}, & \text { put } Z_{t}^{\prime}(\omega)=b_{t} 1_{\left\{t \leq \tau_{2}\right\}}+Z_{t} 1_{\left\{t>\tau_{2}\right\}},\end{cases}
$$


where $(Y, Z)$ is the original bounded solution of the $\operatorname{BSDE}(g, \xi)$.

Thus we get

$$
\begin{aligned}
Z_{t}^{\prime} & =1_{\left\{\tau_{1}<\tau_{2}, \tau_{3}<\alpha_{n+1}\right\}}\left(b_{t} 1_{\left\{t \leq \tau_{1}\right\}}+Z_{t} 1_{\left\{t>\tau_{3}\right\}}\right) \\
& +1_{\left\{\tau_{1}<\tau_{2}, \tau_{3}=\alpha_{n+1}\right\}} b_{t} 1_{\left\{t \leq \tau_{1}\right\}} \\
& +1_{\left\{\tau_{1} \geq \tau_{2}\right\}}\left(b_{t} 1_{\left\{t \leq \tau_{2}\right\}}+Z_{t} 1_{\left\{t>\tau_{2}\right\}}\right) \\
& =1_{\left\{t \leq \tau_{1} \wedge \tau_{2}\right\}} b_{t}+\left[1_{\left\{\tau_{1}<\tau_{2}, \tau_{3}<\alpha_{n+1}, t>\tau_{3}\right\}}+1_{\left\{\tau_{1} \geq \tau_{2}, t>\tau_{2}\right\}}\right] Z_{t} .
\end{aligned}
$$

Obviously, $Z^{\prime}$ is a predictable process.

Set

$$
X_{t} \triangleq X_{\alpha_{n}}+\int_{\alpha_{n}}^{t} g\left(Z_{u}^{\prime}\right) d u-\int_{\alpha_{n}}^{t} Z_{u}^{\prime} d B_{u}
$$

for all $t \in\left[\alpha_{n}, \alpha_{n+1}\right]$.

Step 4. Some properties of $X$.

It follows from the construction that $\left\{X_{t}\right\}_{\alpha_{n} \leq t \leq \alpha_{n+1}}$ has the following properties:

1. $X_{t} 1_{\left\{\tau_{2} \leq \tau_{1}, t \geq \tau_{2}\right\}}=Y_{t} 1_{\left\{\tau_{2} \leq \tau_{1}, t \geq \tau_{2}\right\}}$;

2. $X_{t} 1_{\left\{\tau_{1}<\tau_{2}, \tau_{3} \geq t \geq \tau_{1}\right\}}=\left(X_{\alpha_{n}}-2^{-n-1} \varepsilon\right) 1_{\left\{\tau_{1}<\tau_{2}, \tau_{3} \geq t \geq \tau_{1}\right\}}$;

3. $X_{t} 1_{\left\{\tau_{1}<\tau_{2}, t>\tau_{3}\right\}}=Y_{t} 1_{\left\{\tau_{1}<\tau_{2}, t>\tau_{3}\right\}}$.

Therefore, we have

$$
\begin{aligned}
X_{\alpha_{n+1}} & =Y_{\alpha_{n+1}}\left(1_{\left\{\tau_{2} \leq \tau_{1}\right\}}+1_{\left\{\tau_{1}<\tau_{2}, \tau_{3}<\alpha_{n+1}\right\}}\right) \\
& +\left(X_{\alpha_{n}}-2^{-n-1} \varepsilon\right) 1_{\left\{\tau_{1}<\tau_{2}, \tau_{3}=\alpha_{n+1}\right\}}
\end{aligned}
$$

So the induction assumption $X_{\alpha_{n}} \leq Y_{\alpha_{n}}$ implies $X_{\alpha_{n+1}} \leq Y_{\alpha_{n+1}}$. It is also clear that the new solution $X$ is bounded by $\|Y\|_{\infty}+|y|+\varepsilon$.

Set $A_{n} \triangleq\left\{\omega \in \Omega \mid \tau_{1}<\tau_{2}, \tau_{3}=\alpha_{n+1}\right\}$. Then $P\left(A_{n}\right)$ is the probability that $X_{\alpha_{n+1}}$ is not equal to $Y_{\alpha_{n+1}}$. From (3.10), we get

$P\left(A_{n}\right) \leq P\left(\left\{\omega \in \Omega \mid \exists t \in\left[\alpha_{n}, \alpha_{n+1}\right)\right.\right.$ such that $\left.\left.V_{t}(\omega)<-2^{-n-1} \varepsilon\right\}\right) \leq \exp \left\{-2^{n} \varepsilon\right\}$.

Since $\sum_{n=0}^{\infty} \exp \left\{-2^{n} \varepsilon\right\}<+\infty$, the Borel-Cantelli Lemma implies that

$$
P\left(\cap_{n=0}^{\infty} \cup_{k \geq n} A_{k}\right)=0
$$

which shows $X_{T}=Y_{T}=\xi, \quad P$ a.s. 
To sum up, $\left(X, Z^{\prime}\right)$ is indeed a new bounded solution with $X_{0}=y$.

The construction used many different constants. It is clear that this yields infinitely many different solutions.

Notice that in the proof we only use the fact that $g$ is superquadratic to guarantee that the new solution $X$ is bounded below. This shows if $\mathrm{g}$ is at least quadratic, i.e. $\varlimsup_{|z| \rightarrow \infty} \frac{g(z)}{|z|^{2}}>0$, we can construct a process $V_{t}$ such that $\lim _{t \rightarrow \alpha_{n+1}} V_{t}=+\infty$ as well. Thus we have the following conclusion.

Corollary 3.1. Suppose $g$ is at least quadratic $\overline{\lim }_{|z| \rightarrow \infty} \frac{g(z)}{|z|^{2}}>0$ and, for $\xi \in L^{\infty}\left(\mathcal{F}_{T}\right)$, $Y$ is a bounded solution of the BSDE $(g, \xi)$, then for each $y<Y_{0}$, there exists infinitely many solutions $X$ which are bounded above with $X_{0}=y$.

\subsection{Non-stability of the solutions}

The monotone stability plays an important role in the study of quadratic BSDEs (See, e.g., [12, 3]). Here we shall show that the same type of monotone stability does not hold.

Theorem 3.4. (Non-stability) Suppose $\varlimsup_{z \rightarrow \infty} \frac{g(z)}{|z|^{2}}=\infty$. Then there exists a sequence of solutions $\left\{Y^{k}\right\}_{k=1}^{\infty}$ of BSDEs $\left(g, \xi_{k}\right)$ which increasingly and boundedly converges to $Y$ such that $Y$ is not a solution of $B S D E(g, \xi)$, where $\xi$ is the $L^{\infty}$ limit of $\left\{\xi_{k}\right\}_{k=1}^{\infty}$.

Proof. It follows from $\varlimsup_{z \rightarrow \infty} \frac{g(z)}{|z|^{2}}=\infty$ that there exists a sequence $\left\{z_{k}\right\}_{k=1}^{\infty}$ with $\left|z_{k}\right| \rightarrow+\infty$ such that $g\left(z_{k}\right) \geq \max \left\{16^{k} T\left|z_{k}\right|^{2}, 2^{k+1} T\right\}$. W.l.o.g., we suppose that $z_{k}>0$

Denote $\alpha_{k}:=\left\lceil g\left(z_{k}\right)\right\rceil$ where $\lceil\cdot\rceil$ is the ceiling function and put $Z^{k}(t) \triangleq z_{k} 1_{\cup_{i=1}^{\alpha_{k}}\left[\frac{T}{\alpha_{k}} i-\frac{T}{\alpha_{k}^{2}}, \frac{T}{\alpha_{k}} i\right]}(t)$ for all $0 \leq t \leq T$. Then it follows that

$$
\begin{aligned}
E\left[\int_{0}^{t} Z_{u}^{k} d B_{u}\right]^{2} & \leq \int_{0}^{T}\left(Z_{u}^{k}\right)^{2} d u \\
& =\left(z_{k}\right)^{2} \frac{T}{\alpha_{k}} \leq 16^{-k} \rightarrow 0, \text { for } k \rightarrow \infty .
\end{aligned}
$$

However, we have

$$
\int_{0}^{t} g\left(Z_{u}^{k}\right) d u=\int_{0}^{t} g\left(z_{k}\right) 1_{\cup_{i=1}^{\alpha_{k}}\left[\frac{T}{\alpha_{k}} i-\frac{T}{\alpha_{k}^{2}}, \frac{T}{\alpha_{k}} i\right]}(u) d u \in\left[\frac{g\left(z_{k}\right)}{\alpha_{k}}\left(t-\left(\frac{T}{\alpha_{k}}-\frac{T}{\alpha_{k}^{2}}\right)\right), \frac{g\left(z_{k}\right)}{\alpha_{k}} t\right],
$$


which implies

$$
\sup _{0 \leq t \leq T}\left|\int_{0}^{t} g\left(Z_{u}^{k}\right) d u-t\right| \leq 2^{-k} \rightarrow 0
$$

as $k \rightarrow \infty$.

Define stopping times

$$
\nu_{k} \triangleq \inf \left\{t \geq 0|| \int_{0}^{t} Z_{u}^{k} d B_{u} \mid>2^{-k}\right\} \wedge T
$$

and

$$
\nu=\inf _{k \geq 1} \nu_{k}
$$

Applying the submartingale inequality, we get

$$
\begin{aligned}
P\left[\nu_{k}<T\right] & =P\left[\sup _{0 \leq t \leq T}\left|\int_{0}^{t} Z_{u}^{k} d B_{u}\right|>2^{-k}\right] \\
& \leq 4^{k} E\left[\left(\int_{0}^{T} Z_{u}^{k} d B_{u}\right)^{2}\right] \leq 4^{-k} .
\end{aligned}
$$

Thus we get

$$
\begin{aligned}
P[\nu=T] & =1-P\left[\cup_{k \geq 1}\left\{\nu_{k}<T\right\}\right] \\
& \geq 1-\sum_{k \geq 1} P\left[\nu_{k}<T\right] \\
& \geq \frac{2}{3}>0
\end{aligned}
$$

which is due to the selection of sufficient large $z_{k}, k \geq 1$.

Since $\sum_{k \geq 1} P\left[\nu_{k}<T\right]<\infty$, it follows from the Borel-Cantelli Lemma that

$$
P\left[\cap_{n \geq 1} \cup_{k \geq n}\left\{\nu_{k}<T\right\}\right]=0,
$$

which means $P\left[\cup_{n \geq 1} \cap_{k \geq n}\left\{\nu_{k}=T\right\}\right]=1$. It implies that, for almost all $\omega \in \Omega$, there exists $N(\omega)$ such that for any $k>N(\omega), \nu_{k}(\omega)=T$. Thus we have $P[\nu>0]=1$.

Define $y_{t}^{k}=\int_{0}^{t \wedge \nu} g\left(Z_{u}^{k}\right) d u-\int_{0}^{t \wedge \nu} Z_{u}^{k} d B_{u}$. We then deduce that

$$
\begin{aligned}
& \sup _{0 \leq t \leq T}\left|y_{t}^{k}-t \wedge \nu\right| \\
\leq & \sup _{0 \leq t \leq T}\left|\int_{0}^{t \wedge \nu} g\left(Z_{u}^{k}\right) d u-t \wedge \nu\right|+\sup _{0 \leq t \leq T}\left|\int_{0}^{t \wedge \nu} Z_{u}^{k} d B_{u}\right| \\
\leq & 2 \cdot 2^{-k}
\end{aligned}
$$

which implies that

$$
\lim _{k \rightarrow \infty} \sup _{0 \leq t \leq T}\left|y_{t}^{k}-t \wedge \nu\right|=0
$$


Set

$$
Y_{t}^{n}=y_{t}^{n}-8+\sum_{k=1}^{n} 4 \cdot 2^{-(k-1)} .
$$

Notice that the stopping time $\nu_{k}$ is defined such that $\left|\int_{0}^{t \wedge \nu} Z_{u}^{k} d B_{u}\right| \leq 2^{-k}, \forall t \in$ $[0, T]$. Combining (3.21) with the definition of $\nu_{k}$, we get that

$$
\begin{aligned}
Y_{t}^{k}-Y_{t}^{k-1} & =y_{t}^{k}-y_{t}^{k-1}+4 \cdot 2^{-k} \\
& \geq\left[t \wedge \nu-2 \cdot 2^{-k}\right]-\left[t \wedge \nu+2 \cdot 2^{-(k-1)}\right]+4 \cdot 2^{-(k-1)} \\
& \geq 0
\end{aligned}
$$

which shows that $\left\{Y^{k}\right\}_{k=1}^{\infty}$ is a nondecreasing sequence. Set $\xi^{k}=Y_{T}^{k}$ for $k \geq 1$. Then $Y^{k}$ is a solution of the $\operatorname{BSDE}\left(g, \xi^{k}\right)$. It follows from (3.22) that $Y_{t}^{k}$ converges to $t \wedge \nu$ as $k \rightarrow \infty$ and $\xi^{k} \rightarrow \nu$ in $L^{\infty}$. However, $t \wedge \nu$ is not a solution of the $\operatorname{BSDE}(g, \nu)$ for $t \wedge \nu$ is an increasing process.

Remark 3.4. Although $t \wedge \nu$ is not the solution of the BSDE $(g, \nu)$, it is the dynamic utility function of $\nu$, i.e. $U_{t}(\nu)=t \wedge \nu$.

Proof. Indeed, setting the measure $Q^{k}$ such that $E_{P}\left[\frac{d Q^{k}}{d P} \mid \mathcal{F}_{t}\right]=\mathcal{E}\left(q^{k} \cdot B\right)_{t}$ where $q_{t}^{k}=g^{\prime}\left(z_{k}\right) 1_{\cup_{i=1}^{\alpha_{k}}\left[\frac{T}{\alpha_{k}} i-\frac{T}{\alpha_{k}^{2}}, \frac{T}{\alpha_{k}} i\right]}(t \wedge \nu)$, we have

$$
E\left[\exp \left\{\frac{1}{2} \int_{0}^{T}\left(q_{t}^{k}\right)^{2} d t\right\}\right] \leq \exp \left\{\frac{1}{2}\left(g^{\prime}\left(z_{k}\right)\right)^{2} T\right\}<\infty \text {. }
$$

So $\mathcal{E}\left(q^{k} \cdot B\right)_{t}$ is a $P$-martingale and $Q^{k}$ is well defined. Then

$$
\begin{aligned}
U_{t}\left(\xi^{k}\right) & \leq E_{Q^{k}}\left[\xi^{k}+\int_{t}^{T} f\left(q_{u}^{k}\right) d u \mid \mathcal{F}_{t}\right] \\
& =Y_{t}^{k}+E_{Q^{k}}\left[\int_{t}^{\nu}\left(f\left(q_{u}^{k}\right)+g\left(Z_{u}^{k}\right)-Z_{u}^{k} q_{u}^{k}\right) d u \mid \mathcal{F}_{t}\right] \\
& =Y_{t}^{k} .
\end{aligned}
$$

Thus it follows from Lemma 2.3 that $U_{t}\left(\xi^{k}\right)=Y_{t}^{k}$. If $k$ tends to infinity, we get

$$
U_{t}(\nu)=t \wedge \nu
$$

since $\xi^{k} \rightarrow \nu$ in $L^{\infty}$.

Remark 3.5. $\nu$ is not minimal since $\nu \geq 0$ with $P(\nu>0)>0$ and $U_{0}(\nu)=0$. 


\subsection{A solution to $\operatorname{BSDE}(g, \nu)$}

In the following, we find a bounded solution of $\operatorname{BSDE}(g, \nu)$ where $0<\nu \leq T$ is a stopping time. Of course we can then construct infinitely many bounded solutions for the BSDE.

Step 1. For any $y_{0}<0$, construct an $\mathcal{F}$-predictable process $H$ which can be dominated by $t \wedge \nu(\omega)$ and $t \wedge \nu(\omega)+\left(1-\frac{t}{T}\right) y_{0}$ for any $t$ small enough.

Since $g$ is superquadratic and continuous, we can get an increasing sequence $\left\{x_{i}\right\}_{i=1}^{\infty}$ such that

$$
g\left(x_{i}\right)=i^{2} x_{i}^{2}
$$

for any $i \geq \sqrt{a}+1$ where $a=\inf _{|x|>0} \frac{g(x)}{x^{2}}$ and $x_{i}=1$ for any $i<\sqrt{a}+1$.

Set $k_{t}=x_{i}$ when $t \in\left(\sum_{n=i+1}^{\infty} \delta_{n}, \sum_{n=i}^{\infty} \delta_{n}\right]$ for $i \geq 1$ where $\delta_{n}=\frac{a_{1}}{g\left(x_{n}\right) n^{2}}$, and $a_{1}=\frac{T}{\sum_{n=1}^{\infty} \frac{1}{g\left(x_{n}\right) n^{2}}}$ is such that $\sum_{n=1}^{\infty} \delta_{n}=T$.

We then have

$$
\begin{gathered}
\int_{0}^{T}\left(k_{u}\right)^{2} d u=\sum_{i=1}^{\infty} x_{i}^{2} \frac{a_{1}}{g\left(x_{i}\right) i^{2}}<\infty, \\
\int_{0}^{T} g\left(k_{u}\right) d u=\sum_{i=1}^{\infty} g\left(x_{i}\right) \frac{a_{1}}{g\left(x_{i}\right) i^{2}}<\infty .
\end{gathered}
$$

Put $H_{t}=y_{0}+\int_{0}^{t} g\left(k_{u}\right) d u-\int_{0}^{t} k_{u} d B_{u}$.

Lemma 3.1. There exists $\Omega^{*} \subseteq \Omega$ with $P\left(\Omega^{*}\right)=1$ satisfying for any $\varepsilon>0$ and $\omega \in \Omega^{*}$ there is $t_{\varepsilon}(\omega)$ such that, for any $t<t_{\varepsilon}(\omega), t \wedge \nu(\omega)+\left(1-\frac{t}{T}\right) y_{0}<H_{t}(\omega)$.

Proof. It follows from the law of the iterated logarithm of Brownian motion that there exists a set $\Omega^{*}, P\left(\Omega^{*}\right)=1$ satisfying: for any $\varepsilon>0$ and $\omega \in \Omega^{*}$ there is $t_{\varepsilon}(\omega)<\nu$ such that

$$
\left[\int_{0}^{t} k_{u} d B_{u}\right](\omega) \leq(1+\varepsilon) \sqrt{2\left(\int_{0}^{t} k_{u}^{2} d u\right) \log \log \left(1 / \int_{0}^{t} k_{u}^{2} d u\right)}
$$

for any $t<t_{\varepsilon}(\omega)$.

Set $F(t)=\int_{0}^{t} g\left(k_{u}\right) d u-(1+\varepsilon) \sqrt{2\left(\int_{0}^{t} k_{u}^{2} d u\right) \log \log \left(1 / \int_{0}^{t} k_{u}^{2} d u\right)}-\left(\frac{T-y_{0}}{T}\right) t$. Now we want to prove $F(t)>0$ for $t$ small enough. Calculating the differential of $F$ with respect to $t$, we have, for sufficiently small $t$,

$$
F^{\prime}(t)>g\left(k_{t}\right)-1+\frac{y_{0}}{T}-\gamma k_{t}^{2}\left(\frac{1}{c_{t}} \log \log \frac{1}{c_{t}}\right)^{1 / 2}
$$


where $\gamma=(1+\varepsilon) 2^{-\frac{1}{2}}$ and $c_{t}=\int_{0}^{t} k_{u}^{2} d u$. For $t \in\left(\sum_{n=i+1}^{\infty} \delta_{n}, \sum_{n=i}^{\infty} \delta_{n}\right]$, we get

$$
F^{\prime}(t)>g\left(x_{i}\right)-1+\frac{y_{0}}{T}-\gamma x_{i}^{2}\left(\frac{1}{c_{t}} \log \log \frac{1}{c_{t}}\right)^{1 / 2}
$$

and

$$
\begin{aligned}
c_{t} & \geq \int_{0}^{\sum_{n=i+1}^{\infty} \delta_{n}} k_{u}^{2} d u \\
& =\sum_{n=i+1}^{\infty} x_{n}^{2} \delta_{n} \\
& =a_{1} \sum_{n=i+1}^{\infty} n^{-4},
\end{aligned}
$$

for $i$ big enough. Thus

$$
F^{\prime}(t)>i^{2} x_{i}^{2}-1+\frac{y_{0}}{T}-\gamma x_{i}^{2}\left(\frac{1}{a_{1} \sum_{n=i+1}^{\infty} n^{-4}} \log \log \frac{1}{a_{1} \sum_{n=i+1}^{\infty} n^{-4}}\right)^{1 / 2} .
$$

It follows from

$$
\lim _{i \rightarrow \infty} \frac{i^{2}}{\gamma\left(\frac{1}{a_{1} \sum_{n=i+1}^{\infty} n^{-4}} \log \log \frac{1}{a_{1} \sum_{n=i+1}^{\infty} n^{-4}}\right)^{1 / 2}}=+\infty
$$

that there exist $0<t_{0}<T$ such that for any $t<t_{0}, F^{\prime}(t)>0$. Since $F(0)=0$, we have, for any $t<t_{0}$,

$$
F(t)>0
$$

Thus for any $\omega \in \Omega^{*}$ and $0<t<t_{0} \wedge t_{\varepsilon}(\omega)$, we have

$$
H_{t}(\omega)-\left[t \wedge \nu(\omega)+\left(1-\frac{t}{T}\right) y_{0}\right] \geq F(t)>0 .
$$

Step 2. Since $H_{t}$ and $t \wedge \nu(\omega)$ are $\left\{\mathcal{F}_{t}\right\}_{t \geq 0}$-predictable, we can define stopping times:

$$
\begin{gathered}
\tau_{1}^{1} \triangleq \inf \left\{t>0 \mid H_{t} \leq t \wedge \nu+\left(1-\frac{t}{T}\right) y_{0}\right\} \wedge T, \\
\tau_{1}^{2} \triangleq \inf \left\{t>0 \mid H_{t} \geq t \wedge \nu\right\} \wedge T .
\end{gathered}
$$

Define a random time

$$
\begin{aligned}
\tau_{1} \triangleq 1_{\left\{\tau_{1}^{1}<\tau_{1}^{2}\right\}} \tau_{1}^{1} & +1_{\left\{\tau_{1}^{1} \geq \tau_{1}^{2}\right\}} 1_{\left\{\tau_{1}^{2} \leq \nu+\left(1-\frac{\nu}{T}\right) y_{0}\right\}} \frac{\tau_{1}^{2}-y_{0}}{T-y_{0}} T \\
& +1_{\left\{\tau_{1}^{1} \geq \tau_{1}^{2}\right\}} 1_{\left\{\nu>\tau_{1}^{2}>\nu+\left(1-\frac{\nu}{T}\right) y_{0}\right\}} \frac{\nu-\tau_{1}^{2}+y_{0}}{y_{0}} T \\
& +1_{\left\{\tau_{1}^{1} \geq \tau_{1}^{2}\right\}} 1_{\left\{\tau_{1}^{2} \geq \nu\right\}} T .
\end{aligned}
$$

It is easy to verify that for any $\omega \in\left\{\tau_{1}^{1} \geq \tau_{1}^{2}\right\}, \tau_{1}(\omega) \wedge \nu+\left(1-\frac{\tau_{1}(\omega)}{T}\right) y_{0}=\tau_{1}^{2} \wedge \nu$. 
Lemma 3.2. $\tau_{1}$ is a stopping time.

Proof. This is straightforward but for completeness we give a proof. The random time $\tau_{1}$ is defined by four parts without any intersections. For the first part, it is easily verified by

$$
\left\{1_{\left\{\tau_{1}^{1}<\tau_{1}^{2}\right\}} \tau_{1}^{1} \leq t\right\}=\left\{\tau_{1}^{1}<\tau_{1}^{2}\right\} \cap\left\{\tau_{1}^{1} \leq t\right\} \in \mathcal{F}_{t}
$$

For the second part, it is necessary to check that

$$
\left\{\tau_{1}^{1} \geq \tau_{1}^{2}\right\} \cap\left\{\tau_{1}^{2} \leq \nu+\left(1-\frac{\nu}{T}\right) y_{0}\right\} \cap\left\{\frac{\tau_{1}^{2}-y_{0}}{T-y_{0}} T \leq t\right\} \in \mathcal{F}_{t}
$$

It follows from $t\left(1-\frac{y_{0}}{T}\right)+y_{0} \leq t$ that

$$
\left\{\frac{\tau_{1}^{2}-y_{0}}{T-y_{0}} T \leq t\right\}=\left\{\tau_{1}^{2} \leq t\left(1-\frac{y_{0}}{T}\right)+y_{0}\right\} \in \mathcal{F}_{t}
$$

which implies that $\frac{\tau_{1}^{2}-y_{0}}{T-y_{0}} T$ is a stopping time.

If $\omega \in\left\{\frac{\tau_{1}^{2}-y_{0}}{T-y_{0}} T \leq t\right\}$, then we have

$$
\tau_{1}^{2}(\omega) \leq t+\left(1-\frac{t}{T}\right) y_{0} \leq t
$$

Thus

$$
\begin{aligned}
& \left\{\tau_{1}^{1} \geq \tau_{1}^{2}\right\} \cap\left\{\tau_{1}^{2} \leq \nu+\left(1-\frac{\nu}{T}\right) y_{0}\right\} \cap\left\{\frac{\tau_{1}^{2}-y_{0}}{T-y_{0}} T \leq t\right\} \\
= & \left\{\tau_{1}^{1} \geq \tau_{1}^{2}\right\} \cap\left\{\tau_{1}^{2} \leq t\right\} \cap\left\{\frac{\tau_{1}^{2}-y_{0}}{T-y_{0}} T \leq \nu\right\} \cap\left\{\frac{\tau_{1}^{2}-y_{0}}{T-y_{0}} T \leq t\right\} \\
= & {\left[\left\{\tau_{1}^{1} \geq \tau_{1}^{2}\right\} \cap\left\{\tau_{1}^{2} \leq t\right\}\right] \cap\left\{\frac{\tau_{1}^{2}-y_{0}}{T-y_{0}} T \leq \nu \wedge t\right\} } \\
\in & \mathcal{F}_{t} .
\end{aligned}
$$

For the third part, observe that for any $\omega \in\left\{\tau_{1}^{2}>\nu+\left(1-\frac{\nu}{T}\right) y_{0}\right\} \cap\left\{\frac{\nu-\tau_{1}^{2}+y_{0}}{y_{0}} T \leq t\right\}$, we have

$$
\nu(\omega)-\tau_{1}^{2}(\omega) \geq\left(\frac{t}{T}-1\right) y_{0} \geq 0 .
$$

Combining with $\nu(\omega)-\tau_{1}^{2}(\omega)<\left(\frac{\nu(\omega)}{T}-1\right) y_{0}$, we get

$$
\tau_{1}^{2}(\omega) \leq \nu(\omega)<t
$$


Therefore,

$$
\begin{aligned}
& \left\{\tau_{1}^{1} \geq \tau_{1}^{2}\right\} \cap\left\{\nu>\tau_{1}^{2}>\nu+\left(1-\frac{\nu}{T}\right) y_{0}\right\} \cap\left\{\frac{\nu-\tau_{1}^{2}+y_{0}}{y_{0}} T \leq t\right\} \\
= & \left\{\tau_{1}^{1} \geq \tau_{1}^{2}\right\} \cap\left\{\tau_{1}^{2}>\nu+\left(1-\frac{\nu}{T}\right) y_{0}\right\} \cap\left\{\frac{\nu-\tau_{1}^{2}+y_{0}}{y_{0}} T \leq t\right\} \\
= & \left(\left\{\tau_{1}^{1} \geq \tau_{1}^{2}\right\} \cap\left\{\tau_{1}^{2}<t\right\}\right) \cap\left(\left\{\frac{\tau_{1}^{2}-y_{0}}{T-y_{0}} T>\nu\right\} \cap\{\nu<t\}\right) \\
& \cap\left(\left\{\tau_{1}^{2}+\left(\frac{t}{T}-1\right) y_{0} \leq \nu\right\} \cap\{\nu<t\}\right) \\
\in & \mathcal{F}_{t} .
\end{aligned}
$$

The fourth part is obviously $\mathcal{F}_{t}$-measurable. Thus from $(3.26)$ to $(3.28)$ we get that $\tau_{1}$ is a stopping time.

Define the predictable process $Z$ on the set $\left\{t \leq \tau_{1}\right\}$ as:

$$
Z_{t} 1_{\left\{t \leq \tau_{1}\right\}}=k_{t} 1_{\left\{t \leq \tau_{1}^{1} \wedge \tau_{1}^{2}\right\}}
$$

Lemma 3.3. Set $X_{t} \triangleq y_{0}+\int_{0}^{t} g\left(Z_{u}\right) d u-\int_{0}^{t} Z_{u} d B_{u}$. We have $X_{\tau_{1}}=\tau_{1} \wedge \nu+\left(1-\frac{\tau_{1}}{T}\right) y_{0}$.

Proof. The definitions of the stopping times yield that

$$
\begin{aligned}
X_{\tau_{1}}= & y_{0}+\int_{0}^{\tau_{1}} g\left(Z_{u}\right) d u-\int_{0}^{\tau_{1}} Z_{u} d B_{u} \\
= & y_{0}+\int_{0}^{T} g\left(Z_{u} 1_{\left\{t \leq \tau_{1}\right\}}\right) d u-\int_{0}^{T} Z_{u} 1_{\left\{t \leq \tau_{1}\right\}} d B_{u} \\
= & y_{0}+\int_{0}^{T} g\left(k_{u}\right) 1_{\left\{t \leq \tau_{1}^{1} \wedge \tau_{1}^{2}\right\}} d u-\int_{0}^{T} k_{u} 1_{\left\{t \leq \tau_{1}^{1} \wedge \tau_{1}^{2}\right\}} d B_{u} \\
= & \left(y_{0}+\int_{0}^{\tau_{1}^{1}} g\left(k_{u}\right) d u-\int_{0}^{\tau_{1}^{1}} k_{u} d B_{u}\right) 1_{\left\{\tau_{1}^{1}<\tau_{1}^{2}\right\}} \\
& +\left(y_{0}+\int_{0}^{\tau_{1}^{2}} g\left(k_{u}\right) d u-\int_{0}^{\tau_{1}^{2}} k_{u} d B_{u}\right) 1_{\left\{\tau_{1}^{1} \geq \tau_{1}^{2}\right\}} \\
= & \left(\tau_{1}^{1} \wedge \nu+\left(1-\frac{\tau_{1}^{1}}{T}\right) y_{0}\right) 1_{\left\{\tau_{1}^{1}<\tau_{1}^{2}\right\}}+\left(\tau_{1}^{2} \wedge \nu\right) 1_{\left\{\tau_{1}^{1} \geq \tau_{1}^{2}\right\}} \\
= & \left(\tau_{1} \wedge \nu+\left(1-\frac{\tau_{1}}{T}\right) y_{0}\right) 1_{\left\{\tau_{1}^{1}<\tau_{1}^{2}\right\}}+\left(\tau_{1} \wedge \nu+\left(1-\frac{\tau_{1}}{T}\right) y_{0}\right) 1_{\left\{\tau_{1}^{1} \geq \tau_{1}^{2}\right\}} \\
= & \tau_{1} \wedge \nu+\left(1-\frac{\tau_{1}}{T}\right) y_{0}
\end{aligned}
$$

which completes the proof.

Step 3. Consider the solution in the time interval $\left(\tau_{1}, T\right)$.

Construct $H_{t}^{2}=\tau_{1} \wedge \nu+\left(1-\frac{\tau_{1}}{T}\right) y_{0}+\int_{\tau_{1}}^{t} g\left(k_{u-\tau_{1}}\right) d u-\int_{\tau_{1}}^{t} k_{u-\tau_{1}} d B_{u}$ for any $t>\tau_{1}$. 
Set $t=\tau_{1}+s$ where $s>0$. We have

$$
\begin{aligned}
& H_{t}^{2}-\left(t \wedge \nu+\left(1-\frac{t}{T}\right) y_{0}\right) \\
= & \tau_{1} \wedge \nu+\left(1-\frac{\tau_{1}}{T}\right) y_{0}-\left(t \wedge \nu+\left(1-\frac{t}{T}\right) y_{0}\right)+\int_{\tau_{1}}^{t} g\left(k_{u-\tau_{1}}\right) d u-\int_{\tau_{1}}^{t} k_{u-\tau_{1}} d B_{u} \\
\geq & \int_{0}^{s} g\left(k_{u}\right) d u-\int_{\tau_{1}}^{\tau_{1}+s} k_{u-\tau_{1}} d B_{u}+\frac{s}{T} y_{0}-s .
\end{aligned}
$$

Applying the law of the iterated logarithm of Brownian motion to (3.30), we get that there is a set $\Omega^{*} \in \Omega$ with $P\left(\Omega^{*}\right)=1$ such that for any $\varepsilon>0$ and $\omega \in \Omega^{*}$ there exists a $s_{\varepsilon}(\omega)$ satisfying for all $s<s_{\varepsilon}(\omega)$,

$$
\begin{aligned}
& \int_{0}^{s} g\left(k_{u}\right) d u-\int_{\tau_{1}}^{\tau_{1}+s} k_{u-\tau_{1}} d B_{u}+\frac{s}{T} y_{0}-s \\
\geq & \int_{0}^{s} g\left(k_{u}\right) d u-(1+\varepsilon) \sqrt{2\left(\int_{\tau_{1}}^{\tau_{1}+s} k_{u-\tau_{1}}^{2} d u\right) \log \log \left(1 / \int_{\tau_{1}}^{\tau_{1}+s} k_{u-\tau_{1}}^{2} d u\right)}+\frac{s}{T} y_{0}-s \\
= & \int_{0}^{s} g\left(k_{u}\right) d u-(1+\varepsilon) \sqrt{2\left(\int_{0}^{s} k_{u}^{2} d u\right) \log \log \left(1 / \int_{0}^{s} k_{u}^{2} d u\right)}+\frac{s}{T} y_{0}-s \\
= & F(s) .
\end{aligned}
$$

It follows from (3.24) and (3.30) that

$$
H_{\tau_{1}+s}^{2}(\omega)>\left(\tau_{1}+s\right) \wedge \nu(\omega)+\left(1-\frac{\tau_{1}+s}{T}\right) y_{0}
$$

for all $0<s<s_{\varepsilon}(\omega) \wedge t_{0}$.

Then similarly we can define stopping times:

$$
\begin{gathered}
\tau_{2}^{1} \triangleq \inf \left\{t>\tau_{1} \mid H_{t}^{2} \leq t \wedge \nu+\left(1-\frac{t}{T}\right) y_{0}\right\} \wedge T, \\
\tau_{2}^{2} \triangleq \inf \left\{t>\tau_{1} \mid H_{t}^{2} \geq t \wedge \nu\right\} \wedge T,
\end{gathered}
$$

and a random time

$$
\begin{aligned}
\tau_{2} \triangleq 1_{\left\{\tau_{2}^{1}<\tau_{2}^{2}\right\}} \tau_{2}^{1} & +1_{\left\{\tau_{2}^{2} \leq \tau_{2}^{1}\right\}} 1_{\left\{\tau_{2}^{2} \leq \nu+\left(1-\frac{\nu}{T}\right) y_{0}\right\}} \frac{\tau_{2}^{2}-y_{0}}{T-y_{0}} T \\
& +1_{\left\{\tau_{2}^{2} \leq \tau_{2}^{1}\right\}} 1_{\left\{\nu>\tau_{2}^{2}>\nu+\left(1-\frac{\nu}{T}\right) y_{0}\right\}} \frac{\nu-\tau_{2}^{2}+y_{0}}{y_{0}} T \\
& +1_{\left\{\tau_{2}^{2} \leq \tau_{2}^{1}\right\}} 1_{\left\{\tau_{2}^{2} \geq \nu\right\}} T,
\end{aligned}
$$

which is also a stopping time by a similar proof of $\tau_{1}$ in Lemma 3.2 .

Step 3. Define the random times by transfinite induction.

The random time $\tau_{\alpha}$ for some ordinal number $\alpha$ is defined by the following rules: 
1. $\tau_{0}=0$;

2. If $E\left[\tau_{\alpha}\right]<T$, define $\tau_{\alpha+1}^{1} \triangleq \inf \left\{t>\tau_{\alpha} \mid H_{t}^{\alpha+1} \leq t \wedge \nu+\left(1-\frac{t}{T}\right) y_{0}\right\} \wedge T$,

$$
\begin{aligned}
\tau_{\alpha+1}^{2} \triangleq & \inf \left\{t>\tau_{\alpha} \mid H_{t}^{\alpha+1} \geq t \wedge \nu\right\} \wedge T \text { and } \\
\tau_{\alpha+1} \triangleq 1_{\left\{\tau_{\alpha+1}^{1}<\tau_{\alpha+1}^{2}\right\}} \tau_{\alpha+1}^{1} & +1_{\left\{\tau_{\alpha+1}^{2} \leq \tau_{\alpha+1}^{1}\right\}} 1_{\left\{\tau_{\alpha+1}^{2} \leq \nu+\left(1-\frac{\nu}{T}\right) y_{0}\right\}} \frac{\tau_{\alpha+1}^{2}-y_{0}}{T-y_{0}} T \\
& +1_{\left\{\tau_{\alpha+1}^{2} \leq \tau_{\alpha+1}^{1}\right\}} 1_{\left\{\nu>\tau_{\alpha+1}^{2}>\nu+\left(1-\frac{\nu}{T}\right) y_{0}\right\}} \frac{\nu-\tau_{\alpha+1}^{2}+y_{0}}{y_{0}} T \\
& +1_{\left\{\tau_{\alpha+1}^{2} \leq \tau_{\alpha+1}^{1}\right\}} 1_{\left\{\tau_{\alpha+1}^{2} \geq \nu\right\}} T
\end{aligned}
$$

where $H_{t}^{\alpha+1}=\tau_{\alpha} \wedge \nu+\left(1-\frac{\tau_{\alpha}}{T}\right) y_{0}+\int_{\tau_{\alpha}}^{t} g\left(k_{u-\tau_{\alpha}}\right) d u-\int_{\tau_{\alpha}}^{t} k_{u-\tau_{\alpha}} d B_{u}$ for $t>\tau_{\alpha}$.

3. If $\beta$ is a limit number and satisfies $E\left[\tau_{\alpha}\right]<T$, for all $\alpha<\beta$, then $\tau_{\beta} \triangleq \lim _{\alpha<\beta} \tau_{\alpha}$. We adopt the symbol $\omega_{1}$ for the first uncountable ordinal and let $\mathcal{O}$ be the well ordered set of all countable ordinals, i.e. ordinals $\alpha<\omega_{1}$. Define

$$
\Lambda \triangleq\left\{\alpha \in \mathcal{O} \mid E\left[\tau_{\xi}\right]<T \text {, for all } \xi<\alpha\right\}
$$

Since $\left\{E\left[\tau_{\alpha}\right]\right\}_{\alpha \in \Lambda}$ is strictly increasing, $\Lambda$ is countable and hence there must exist $\beta_{0}$ with $E\left[\tau_{\beta_{0}}\right]=T$, hence $\tau_{\beta_{0}}=T$.

Define the predictable process $Z$ by

$$
Z_{t}=\sum_{0 \leq k<\beta_{0}} k_{t-\tau_{k}} 1_{\left\{\tau_{k}<t \leq \tau_{k}+\tau_{k+1}^{1} \wedge \tau_{k+1}^{2}\right\}}
$$

and the stochastic process

$$
X_{t}=y_{0}+\int_{0}^{t} g\left(Z_{u}\right) d u-\int_{0}^{t} Z_{u} d B_{u}
$$

for any $t \in[0, T]$.

Similarly as lemma (3.3), we have

$$
X_{\tau_{k}}=\tau_{k} \wedge \nu+\left(1-\frac{\tau_{k}}{T}\right) y_{0}
$$

for any $k<\beta_{0}$. Letting $k$ tend to $\beta_{0}$, we get $X_{T}=\nu$.

Therefore, we constructed a solution $(X, Z)$ for the $\operatorname{BSDE}(g, \nu)$ with $X_{0}=y_{0}<0$. 
Example 3.1. In this example, our goal is to construct a bounded random variable $\zeta$ such that $U(\zeta)$ is a solution of $\operatorname{BSDE}(g, \zeta)$ and $\zeta$ is not minimal when $g$ is superquadratic. Define $\zeta=H_{\tau_{1}^{1} \wedge \tau_{1}^{2}}$, then $\left(H_{t}^{\tau_{1}^{1} \wedge \tau_{1}^{2}}, k_{t} 1_{\left\{t \leq \tau_{1}^{1} \wedge \tau_{1}^{2}\right\}}\right)_{0 \leq t \leq T}$ is a solution to the $B S D E(g, \zeta)$. It follows that

$$
U(\zeta)=H^{\tau_{1}^{1} \wedge \tau_{1}^{2}}
$$

Indeed, for any $t \in(0, T]$, set the probability measure $Q^{t}$ via $\frac{d Q^{t}}{d P}=\mathcal{E}\left(q^{t} \cdot B\right)_{T}$ with $q_{s}^{t}=g^{\prime}\left(k_{s}\right) 1_{\left\{t<s<\tau_{1}^{1} \wedge \tau_{1}^{2}\right\}}$. We then have

$$
E\left[\exp \left(\frac{1}{2} \int_{0}^{T}\left|q_{s}^{t}\right|^{2} d s\right)\right] \leq \exp \left(\frac{1}{2} \int_{t}^{T}\left|g^{\prime}\left(k_{s}\right)\right|^{2} d s\right)<\infty,
$$

which implies $Q^{t} \sim P$. We deduce that

$$
U_{t}(\zeta)=E_{Q^{t}}\left[\zeta+\int_{t}^{T} f\left(q_{s}^{t}\right) d s \mid \mathcal{F}_{t}\right]=H_{t}^{\tau_{1}^{1} \wedge \tau_{1}^{2}}, \text { for any } t \in(0, T]
$$

by the same argument of (3.5). Since $H^{\tau_{1}^{1} \wedge \tau_{1}^{2}}$ is continuous and $U .(\zeta)$ is càdlàg, we get $U_{0}(\zeta)=y_{0}$.

However, $\zeta$ is not minimal since $\zeta \geq y_{0}$ with $P\left(\zeta>y_{0}\right)>0$ and $U_{0}\left(y_{0}\right)=y_{0}$.

\section{Existence of solution to BSDEs in the Marko- vian case}

From the last section, we know that the BSDE with superquadratic growth is ill-posed. However we will show that in some particular Markovian case, there exists a solution for such a BSDE.

Define the diffusion process $X^{t, x}$ to be the solution to the following SDE:

$$
\begin{aligned}
d X_{s} & =b\left(s, X_{s}\right) d s+\sigma d B_{s}, \quad t \leq s \leq T \\
X_{s} & =x, \quad 0 \leq s \leq t
\end{aligned}
$$

where $b:[0, T] \times \mathbb{R}^{n} \rightarrow \mathbb{R}^{n}$ is continuously differentiable with respect to $x$ with bounded derivative $b_{x}$, and $\sigma:[0, T] \rightarrow \mathbb{R}^{n \times d}$ is a constant (matrix).

Let us consider BSDE (3.1) with $\xi=\Phi\left(X_{T}^{t, x}\right)$ :

$$
Y_{s}=\Phi\left(X_{T}^{t, x}\right)-\int_{s}^{T} g\left(Z_{r}\right) d r+\int_{s}^{T} Z_{r} d B_{r}, \quad s \in[0, T],
$$


where $g: R^{d} \rightarrow R_{+}$is a continuously differentiable convex function with $g(0)=0$. We suppose it is superquadratic $\varlimsup_{|z| \rightarrow \infty} \frac{g(z)}{|z|^{2}}=\infty . f: R^{d} \rightarrow R_{+} \cup\{\infty\}$ is the FenchelLegendre transform of $g$ :

$$
f(x)=\sup _{z \in R^{d}}(z x-g(z))
$$

then $f$ is also convex and $f(0)=0$.

\subsection{Lipschitz case}

Let us first consider the case when $\Phi$ is sufficiently smooth.

Theorem 4.1. Suppose that $\Phi$ is bounded and Lipschitz. Then there exists a unique solution $\left(Y^{t, x}, Z^{t, x}\right)$ to BSDE (4.1) such that both processes $Y^{t, x}$ and $Z^{t, x}$ are bounded. Furthermore, the solution is a dynamic utility function of the following form

$$
\begin{aligned}
& Y_{s}^{t, x} \\
= & \inf \left\{E_{Q}\left[\Phi\left(X_{T}^{t, x}\right)+\int_{s}^{T} f\left(q_{u}\right) d u \mid \mathcal{F}_{s}\right] \mid Q \sim P\right\} \\
= & \inf \left\{E_{Q}\left[\Phi\left(X_{T}^{t, x}\right)+\int_{s}^{T} f\left(q_{u}\right) d u \mid \mathcal{F}_{s}\right] \mid Q \sim P,\right. \\
& \left.E_{Q}\left[\int_{r}^{T} f\left(q_{u}\right) d u \mid \mathcal{F}_{r}\right] \leq 2\|\Phi\|_{\infty}, \forall r \in[0, T]\right\}
\end{aligned}
$$

for any $s \in[0, T]$.

Proof. First, let us suppose that $\Phi \in \mathcal{C}^{1}$ and that $\Phi_{x}$ is bounded. We apply a truncation argument to prove the existence of solution. Let us introduce the truncation function: for an integer $N, \rho_{N}: \mathbb{R}^{1 \times d} \rightarrow \mathbb{R}^{+}$is smooth, such that $\forall|z| \leq N$, $\rho_{N}(z)=1$; and $\forall|z| \geq N+1, \rho_{N}(z)=0$. Then it is obvious that $\rho_{N} g$ is a bounded Lipschitz function. Hence for any $N$, there exists a unique solution $\left(Y^{N ; t, x}, Z^{N ; t, x}\right)$ to the following BSDE:

$$
Y_{s}=\Phi\left(X_{T}^{t, x}\right)-\int_{s}^{T}\left(\rho_{N} g\right)\left(Z_{r}\right) d r+\int_{s}^{T} Z_{r} d B_{r}
$$

On the other hand, we denote by $\left(F^{N ; t, x}, V^{N ; t, x}\right)$ the unique solution to the following BSDE:

$$
F_{s}=\Phi_{x}\left(X_{T}^{t, x}\right) \nabla_{x} X_{T}^{t, x}-\int_{s}^{T}\left(\rho_{N} g\right)_{z}\left(Z_{r}^{N ; t, x}\right) V_{r} d r+\int_{s}^{T} V_{r} d B_{r}
$$


where $\int_{s}^{T} V_{r} d B_{r}$ means

$$
\sum_{1 \leq i \leq d} \int_{s}^{T} V_{r}^{i} d B_{r}^{i},
$$

with $V^{i}$ denoting the $i$-th line of the $d \times n$ matrix process $V$.

We then have (see, e.g., [14]):

$$
Z_{s}^{N ; t, x}=-F_{s}^{N ; t, x}\left(\nabla_{x} X_{s}^{t, x}\right)^{-1} \sigma .
$$

As for any $N,\left(\rho_{N} g\right)_{z}\left(Z^{N ; t, x}\right)$ is bounded, we can apply a Girsanov transformation to get:

$$
F_{s}^{N ; t, x}=\Phi_{x}\left(X_{T}^{t, x}\right) \nabla_{x} X_{T}^{t, x}+\int_{s}^{T} V_{r}^{N ; t, x} d B_{r}^{N ; t, x},
$$

where $B^{N ; t, x}$ is a Brownian Motion under an equivalent probability measure $Q^{N ; t, x}$. Taking the conditional expectation with respect to the measure $Q^{N ; t, x}$, one finally deduces that

$$
\left|F_{s}^{N ; t, x}\right| \leq\left\|\Phi_{x}\right\|_{\infty} \cdot\left\|\nabla_{x} X_{T}^{t, x}\right\|_{\infty}
$$

which implies that

$$
\begin{aligned}
\left|Z_{s}^{N ; t, x}\right| & =\left|F_{s}^{N ; t, x}\left(\nabla_{x} X_{s}^{t, x}\right)^{-1} \sigma\right| \\
& \leq\|\sigma\| \cdot\left\|\Phi_{x}\right\|_{\infty} \cdot e^{2\left\|b_{x}\right\|_{\infty} T} \\
& :=c .
\end{aligned}
$$

The same argument (recall that $g(0)=0$ ) gives us also that

$$
\left|Y_{s}^{N ; t, x}\right| \leq\|\Phi\|_{\infty}
$$

Taking $N \geq c$, then the solution $\left(Y^{N ; t, x}, Z^{N ; t, x}\right)$ to BSDE (4.3) is actually a solution to BSDE (4.1).

In the case when $\Phi$ is bounded and Lipschitz, we can also prove, by a standard approximation, that there exists a bounded solution $\left(Y^{t, x}, Z^{t, x}\right)$ with $\left|Z^{t, x}\right| \leq c$ with $c=\|\sigma\| \cdot L_{\Phi} \cdot e^{2\left\|b_{x}\right\|_{\infty} T}$ where $L_{\Phi}$ is the Lipschitz constant of $\Phi$.

It is routine to prove the uniqueness of the bounded solution $\left(Y^{t, x}, Z^{t, x}\right)$ where $Z^{t, x}$ is also bounded.

Finally, as $g_{z}\left(Z^{t, x}\right)$ is bounded, and

$$
E_{Q^{N ; t, x}}\left[\int_{r}^{T} f\left(g_{z}\left(Z_{u}^{t, x}\right)\right) d u \mid \mathcal{F}_{r}\right] \leq 2\|\Phi\|_{\infty}, \text { for } N \geq c, \forall r \in[0, T] .
$$


We conclude that $Y^{t, x}$ is a dynamic utility function of the form (4.2).

Remark 4.1. A new solution $(Y, Z)$ can be constructed by the same technique as before with the process $Z$ unbounded.

We define

$$
u(t, x):=Y_{t}^{t, x}
$$

where $\left(Y^{t, x}, Z^{t, x}\right)$ is the unique bounded solution to (4.1) with $Z^{t, x}$ bounded. Since $\Phi$ is Lipschitz, $\left(Y^{t, x}, Z^{t, x}\right)$ is also the unique bounded solution to (4.3) with $N \geq c$. An important property is that $u(t, x)$ is deterministic.

Remark 4.2. It follows from the classical result of Markovian BSDEs that

$$
Y_{s}^{t, x}=Y_{t}^{t, x}, Z_{s}^{t, x}=0, \text { for } s<t
$$

Besides, $\left(Y^{t, x}, Z^{t, x}\right)$ has the Markov property:

$$
Y_{s}^{t, x}=u\left(s, X_{s}^{t, x}\right), \text { for } s \geq t
$$

Furthermore, we have a uniqueness, a stability theorem and a strict comparison theorem for the BSDEs. Thus we get the following proposition.

Proposition 4.1. Suppose that $\Phi$ is bounded and Lipschitz, then $u(t, x)$ defined by (4.8) is bounded and continuous on $[0, T] \times \mathbb{R}^{n}$ and a viscosity solution to the PDE:

$$
\left\{\begin{array}{l}
u_{t}(t, x)+\frac{1}{2} \operatorname{trace}\left(\sigma \sigma^{T} u_{x x}(t, x)\right)+u_{x}(t, x) b(t, x)-g\left(-u_{x}(t, x) \sigma\right)=0 \\
u(T, x)=\Phi(x)
\end{array}\right.
$$

\subsection{A Priori estimates of $Z$}

Now we suppose that both $\Phi$ and $\Phi_{x}$ are bounded. Let us first suppose that $b \equiv 0$ and $n=d, \sigma$ is the identity to explain our main idea. In this case, $X_{T}^{t, x}=x+B_{T}-B_{t}$. Then equation (4.5) turns out to be

$$
Z_{s}^{N ; t, x}=-F_{s}^{N ; t, x}
$$

On the other hand, BSDE (4.4) becomes:

$$
\left\{\begin{array}{l}
d Z_{s}^{N ; t, x}=-\left(\rho_{N} g\right)_{z}\left(Z_{s}^{N ; t, x}\right) V_{s}^{N ; t, x} d s+V_{s}^{N ; t, x} d B_{s}, \quad 0 \leq s \leq T \\
Z_{T}^{N ; t, x}=-\Phi_{x}\left(x+B_{T}-B_{t}\right) .
\end{array}\right.
$$


This gives the following framework (taking $N \geq\left\|\Phi_{x}\right\|_{\infty}$ ):

$$
\left\{\begin{array}{l}
d Y_{s}=g\left(Z_{s}\right) d s-Z_{s} d B_{s} \\
d Z_{s}=-g_{z}\left(Z_{s}\right) V_{s} d s+V_{s} d B_{s} \\
Y_{T}=\xi \in L^{\infty}\left(\mathcal{F}_{T}\right), \quad Y \text { bounded }
\end{array}\right.
$$

where $E\left[\int_{0}^{T}\left|Z_{r}\right|^{2} d r\right]<+\infty$ and $\int_{0}^{T}\left|V_{r}\right|^{2} d r<+\infty \quad P$ a.s. Thus we get special second order backward stochastic differential equations (see [5] for a definition).

Theorem 4.2. In the framework (4.11), suppose there is a solution and

1) The probability measure $Q$ with $\frac{d Q}{d P}=\mathcal{E}\left(g_{z}(Z) B\right)_{T}$ is equivalent to $P$;

2) $Z$ is a $Q$-martingale.

We then have

$$
\left|Z_{s}\right| \leq 2\|\xi\|_{\infty}(T-s)^{-\frac{1}{2}}, \quad \forall s \in[0, T)
$$

Furthermore, if $f\left(g_{z}(\cdot)\right): \mathbb{R}^{d} \rightarrow \mathbb{R}^{+}$is convex, we also have:

$$
f\left(g_{z}\left(Z_{s}\right)\right) \leq 2\|\xi\|_{\infty}(T-s)^{-1}, \quad \forall s \in[0, T) .
$$

Proof. Under the measure $Q$, we get

$$
d Y_{s}=-f\left(g_{z}\left(Z_{s}\right)\right) d s-Z_{s} d B_{s}^{Q}
$$

where $B_{s}^{Q}=B_{s}-\int_{0}^{s} g_{z}^{T}\left(Z_{r}\right) d r$ is a $Q$-Brownian Motion.

Since $Y$ is bounded and $\int_{0}^{s} f\left(g_{z}\left(Z_{r}\right)\right) d r$ is an increasing process, it follows from Lemma 2.2 that $\int_{0}^{s} Z_{r} d B_{r}^{Q}$ is a BMO martingale under the measure $Q$ :

$$
E_{Q}\left[\int_{s}^{T}\left|Z_{r}\right|^{2} d r \mid \mathcal{F}_{s}\right] \leq 4\|\xi\|_{\infty}^{2}
$$

which implies, by Jensen's inequality,

$$
\left|Z_{s}\right|^{2}(T-s) \leq 4\|\xi\|_{\infty}^{2}
$$

i.e.

$$
\left|Z_{s}\right| \leq 2\|\xi\|_{\infty}(T-s)^{-\frac{1}{2}}
$$

It follows from equation (4.14) that

$$
\begin{aligned}
& E_{Q}\left[\int_{s}^{T} f\left(g_{z}\left(Z_{r}\right)\right) d r \mid \mathcal{F}_{s}\right] \\
= & -E_{Q}\left[\xi-Y_{s} \mid \mathcal{F}_{s}\right] \\
\leq & 2\|\xi\|_{\infty} .
\end{aligned}
$$


If $f\left(g_{z}(\cdot)\right)$ is convex, then applying Jensen's inequality, we get

$$
f\left(g_{z}\left(Z_{s}\right)\right) \leq 2\|\xi\|_{\infty}(T-s)^{-1}
$$

In fact the condition 1) in the theorem is a constraint to make the process $\left\{Z_{t}\right\}_{0 \leq t \leq T}$ not grow so fast as we constructed in the non-uniqueness theorem. In this case, the solution is unique. We have the following remark.

Remark 4.3. Suppose there is a bounded solution $Y$ and the probability measure $Q$ with $\frac{d Q}{d P}=\mathcal{E}\left(g_{z}(Z) B\right)_{T}$ is equivalent to $P$, then the solution is unique and

$$
Y_{s}=E_{Q}\left[\xi+\int_{s}^{T} f\left(g_{z}\left(Z_{r}\right)\right) d r \mid \mathcal{F}_{s}\right], \quad 0 \leq s \leq T .
$$

Let us consider the original BSDE (4.1) again. Taking $N \geq c$ as in the proof of Theorem 4.1, we deduce the following "general" framework:

$$
\begin{cases}d Y_{s}=g\left(Z_{s}\right) d s-Z_{s} d B_{s}, & Y_{T}=\Phi\left(X_{T}\right) \\ d F_{s}=g_{z}\left(Z_{s}\right) V_{s} d s-V_{s} d B_{s}, & F_{T}=\Phi_{x}\left(X_{T}\right) \nabla_{x} X_{T} \\ Z_{s}=-F_{s}\left(\nabla_{x} X_{s}\right)^{-1} \sigma, & \end{cases}
$$

where $\Phi$ and $\Phi_{x}$ are bounded. Under the probability measure $Q, B_{s}^{Q}=B_{s}-\int_{0}^{s} g_{z}\left(Z_{r}\right) d r$ is a Brownian Motion, and the "general" framework becomes:

$$
\begin{cases}d Y_{s}=-f\left(g_{z}\left(Z_{s}\right)\right) d s-Z_{s} d B_{s}^{Q}, & Y_{T}=\Phi\left(X_{T}\right) \\ d F_{s}=-V_{s} d B_{s}^{Q}, & F_{T}=\Phi_{x}\left(X_{T}\right) \nabla_{x} X_{T} \\ Z_{s}=-F_{s}\left(\nabla_{x} X_{s}\right)^{-1} \sigma . & \end{cases}
$$

Recall that

$$
d \nabla_{x} X_{s}=b_{x}\left(X_{s}\right) \nabla_{x} X_{s} d s
$$

from which we deduce

$$
d\left(\nabla_{x} X_{s}\right)^{-1}=-\left(\nabla_{x} X_{s}\right)^{-1}\left(d \nabla_{x} X_{s}\right)\left(\nabla_{x} X_{s}\right)^{-1}=-\left(\nabla_{x} X_{s}\right)^{-1} b_{x}\left(X_{s}\right) d s
$$

Applying Itô's formula, we deduce

$$
d Z_{s}=-\left(d F_{s}\right)\left(\nabla_{x} X_{s}\right)^{-1} \sigma+F_{s}\left(\nabla_{x} X_{s}\right)^{-1} b_{x}\left(X_{s}\right) \sigma d s
$$

We suppose that there exists a constant $\lambda \geq 0$ such that

$$
\forall \eta \in \mathbb{R}^{n},\left|\eta^{T} \sigma \sigma^{T} b_{x}^{T}(x) \eta\right| \leq \lambda\left|\eta^{T} \sigma\right|^{2}
$$


We then have

$$
\begin{aligned}
d\left(\exp (\lambda s) Z_{s}\right) & =\lambda \exp (\lambda s) Z_{s} d s+\exp (\lambda s) F_{s}\left(\nabla_{x} X_{s}\right)^{-1} b_{x}\left(X_{s}\right) \sigma d s+d M_{s} \\
& =F_{s}^{*}\left(\lambda I-b_{x}\left(X_{s}\right)\right) \sigma d s+d M_{s}
\end{aligned}
$$

where $M$ is a $Q$-martingale and

$$
F_{s}^{*}=-\exp (\lambda s) F_{s}\left(\nabla X_{s}\right)^{-1}
$$

Finally,

$$
d\left|\exp (\lambda s) Z_{s}\right|^{2}=d\langle M\rangle_{s}+2\left[\lambda\left|F_{s}^{*} \sigma\right|^{2}-F_{s}^{*} \sigma \sigma^{T} b_{x}^{T}\left(X_{s}\right)\left(F_{s}^{*}\right)^{T}\right] d s+d M_{s}^{*},
$$

where $M^{*}$ is a $Q$-martingale, hence $\left|\exp (\lambda s) Z_{s}\right|^{2}$ is a $Q$-submartingale.

Proposition 4.2. Let us suppose that $\Phi$ is bounded and Lipschitz, $b$ and $\sigma$ satisfy the assumption (4.17), and $\left(Y^{t, x}, Z^{t, x}\right)$ is the unique bounded solution to BSDE (4.1). Then there exists a constant $c_{1}>0$ such that

$$
\left|Z_{s}^{t, x}\right| \leq c_{1}\|\Phi\|_{\infty}(T-s)^{-\frac{1}{2}}, \quad \forall s \in[0, T)
$$

Proof. First let us consider the smooth case when $\Phi$ and $\Phi_{x}$ are bounded. Since $Z^{t, x}$ is of the framework (4.16), from Lemma 2.2, we have

$$
E_{Q}\left[\int_{s}^{T}\left|Z_{r}^{t, x}\right|^{2} d r \mid \mathcal{F}_{s}\right] \leq 4\|\Phi\|_{\infty}^{2}
$$

from which we deduce that

$$
E_{Q}\left[\int_{s}^{T} \exp (2 \lambda r)\left|Z_{r}^{t, x}\right|^{2} d r \mid \mathcal{F}_{s}\right] \leq 4 \exp (2 \lambda T)\|\Phi\|_{\infty}^{2} .
$$

As $\exp (2 \lambda s)\left|Z_{s}^{t, x}\right|^{2}$ is a $Q$-submartingale, it follows that

$$
\exp (2 \lambda s)\left|Z_{s}^{t, x}\right|^{2}(T-s) \leq 4 \exp (2 \lambda T)\|\Phi\|_{\infty}^{2}
$$

i.e.

$$
\left|Z_{s}^{t, x}\right| \leq c_{1}\|\Phi\|_{\infty}(T-s)^{-\frac{1}{2}}
$$

where $c_{1}=2 \exp (\lambda T)$.

We can get the same estimate by a standard approximation when $\Phi$ is only bounded and Lipschitz. 
Remark 4.4. As an example, let us take $g(z)=|z|^{q}$ for $q \geq 2$. Since $\left|\exp (\lambda s) Z_{s}^{t, x}\right|^{2}$ is a Q-submartingale, it is clear that $\left|\exp (\lambda s) Z_{s}^{t, x}\right|^{q}$ is also a $Q$-submartingale for $q \geq 2$. It follows from (4.14) that

$$
\left|Z_{s}^{t, x}\right|^{q} \leq C_{q}\|\Phi\|_{\infty}(T-s)^{-1}, s \in[t, T)
$$

where $C_{q}>0$ is a constant depending only on $q$ and $\lambda$. Suppose $u$ is the bounded classical solution to the following PDE:

$$
\left\{\begin{array}{l}
u_{t}(t, x)+\frac{1}{2} \operatorname{trace}\left(\sigma \sigma^{T} u_{x x}(t, x)\right)+u_{x}(t, x) b(t)-\left|u_{x}(t, x) \sigma\right|^{q}=0 \\
u(T, x)=\Phi(x)
\end{array}\right.
$$

Since

$$
Z_{s}^{t, x}=-u_{x}\left(s, X_{s}^{t, x}\right)\left(\nabla_{x} X_{s}^{t, x}\right)^{-1} \sigma, \forall s \in[t, T]
$$

we deduce that

$$
\left|u_{x}(t, x) \sigma\right| \leq\left(C_{q}\|\Phi\|_{\infty}\right)^{1 / q}(T-t)^{-1 / q}
$$

The same type of estimate is given by Gilding et al. in [10] using Bernstein's technique, in the case when $b=0$ and $\sigma$ is the identity.

\subsection{Lower semi-continuous case}

Notice that $Z^{t, x}$ is bounded when $\Phi$ is bounded and Lipschitz. The bound, however, depends on the Lipschitz constant. The advantage of the estimate in Proposition 4.2 is that the estimate only depends on $\|\Phi\|_{\infty}$. This allows us to weaken the hypothesis further.

Proposition 4.3. Let us suppose that $\Phi$ is bounded and lower semi-continuous, and $b$ and $\sigma$ satisfy the assumption (4.17). Then there exists a bounded solution $\left(\underline{Y}^{t, x}, \underline{Z}^{t, x}\right)$ to BSDE (4.1) such that

$$
\left|\underline{Z}_{s}^{t, x}\right| \leq c_{1}\|\Phi\|_{\infty}(T-s)^{-\frac{1}{2}}, \quad \forall s \in[t, T) .
$$

Proof. For each integer $m \geq 0$, construct the function

$$
\underline{\Phi}_{m}(u)=\inf \left\{\Phi(p)+m|p-u|: p \in \mathbb{R}^{n}\right\} .
$$


Then $\underline{\Phi}_{m}$ is well defined and globally Lipschitz with Lipschitz constant $m$. Moreover, $\left(\underline{\Phi}_{m}\right)_{m \geq 0}$ is increasing and converges pointwise to $\Phi$ with

$$
-\|\Phi\|_{\infty} \leq \underline{\Phi}_{m} \leq \Phi
$$

Let $\left(\underline{Y}^{m ; t, x}, \underline{Z}^{m ; t, x}\right)$ be the bounded solution to BSDE $\left(g, \underline{\Phi}_{m}\left(X_{T}^{t, x}\right)\right)$. It follows from the classical comparison theorem that

$$
-\|\Phi\|_{\infty} \leq \underline{Y}^{0 ; t, x} \leq \underline{Y}^{m ; t, x} \leq \underline{Y}^{m+1 ; t, x} \leq\|\Phi\|_{\infty}
$$

and from Proposition 4.2 ,

$$
\left|\underline{Z}_{s}^{m ; t, x}\right| \leq c_{1}\|\Phi\|_{\infty}(T-s)^{-\frac{1}{2}}, \quad s \in[0, T)
$$

For any fixed $T^{\prime} \in(0, T),\left(\underline{Y}^{m ; t, x}, \underline{Z}^{m ; t, x}\right)$ satisfies

$$
\underline{Y}_{s}^{m ; t, x}=\underline{Y}_{T^{\prime}}^{m ; t, x}-\int_{s}^{T^{\prime}}\left(\rho_{M} g\right)\left(\underline{Z}_{r}^{m ; t, x}\right) d r+\int_{s}^{T^{\prime}} \underline{Z}_{r}^{m ; t, x} d B_{r}, \quad \forall s \in\left[0, T^{\prime}\right],
$$

where

$$
M=c_{1}\|\Phi\|_{\infty}\left(T-T^{\prime}\right)^{-\frac{1}{2}}
$$

Moreover, by Lemma 2.2,

$$
E\left[\int_{s}^{T}\left|\underline{Z}_{r}^{m ; t, x}\right|^{2} d r \mid \mathcal{F}_{s}\right] \leq 4\|\Phi\|_{\infty}^{2}
$$

The classical stability theorem (see N. El Karoui et al [8]) for Lipschitz generators implies

$$
\lim _{m, m^{\prime} \rightarrow \infty} E\left[\int_{0}^{T^{\prime}}\left|\underline{Z}_{r}^{m ; t, x}-\underline{Z}_{r}^{m^{\prime} ; t, x}\right|^{2} d r\right]=0
$$

So define

$$
\underline{Y}^{t, x}=\lim _{m \rightarrow \infty} \underline{Y}^{m ; t, x}, \quad \underline{Z}^{t, x}=\lim _{m \rightarrow \infty} \underline{Z}^{m ; t, x} .
$$

Then by passing to the limit when $m \rightarrow \infty$ in (4.22), we conclude that for any fixed $T^{\prime} \in(0, T),\left(\underline{Y}^{t, x}, \underline{Z}^{t, x}\right)$ satisfies

$$
Y_{s}=Y_{T^{\prime}}-\int_{s}^{T^{\prime}} g\left(Z_{r}\right) d r+\int_{s}^{T^{\prime}} Z_{r} d B_{r}, \quad \forall s \in\left[0, T^{\prime}\right],
$$

and

$$
\left|\underline{Y}_{s}^{t, x}\right| \leq\|\Phi\|_{\infty}, \quad E\left[\int_{s}^{T}\left|\underline{Z}_{r}^{t, x}\right|^{2} d r \mid \mathcal{F}_{s}\right] \leq 4\|\Phi\|_{\infty}^{2}, \quad s \in[0, T] .
$$


On the other hand, we have

$$
\varliminf_{s \rightarrow T} \underline{Y}_{s}^{t, x} \geq \varliminf_{s \rightarrow T} \underline{Y}_{s}^{m ; t, x}=\underline{\Phi}_{m}\left(X_{T}^{t, x}\right) \text { for any } m \in \mathbb{N}, P \text { a.s. }
$$

which implies $\underline{\lim }_{s \rightarrow T} \underline{Y}_{s}^{t, x} \geq \Phi\left(X_{T}^{t, x}\right)$, P a.s.

Since $\underline{\Phi}_{m}$ is bounded and Lipschitz for any $m \in \mathbb{N}$, it follows from Theorem 4.1 that $\underline{Y}_{s}^{m ; t, x} \leq E\left[\underline{\Phi}_{m}\left(X_{T}^{t, x}\right) \mid \mathcal{F}_{s}\right]$. We then get

$$
\begin{aligned}
\varlimsup_{s \rightarrow T} \underline{Y}_{s}^{t, x} & =\varlimsup_{s \rightarrow T} \lim _{m \rightarrow \infty} \underline{Y}_{s}^{m ; t, x} \\
& \leq \varlimsup_{s \rightarrow T} \lim _{m \rightarrow \infty} E\left[\underline{\Phi}_{m}\left(X_{T}^{t, x}\right) \mid \mathcal{F}_{s}\right] \\
& =\varlimsup_{s \rightarrow T} E\left[\Phi\left(X_{T}^{t, x}\right) \mid \mathcal{F}_{s}\right] \\
& =\Phi\left(X_{T}^{t, x}\right), \quad P \text { a.s. }
\end{aligned}
$$

Hence $\lim _{s \rightarrow T} \underline{Y}_{s}^{t, x}=\Phi\left(X_{T}^{t, x}\right)$.

Finally, passing to the limit when $T^{\prime} \rightarrow T$ in (4.23), we conclude that $\left(\underline{Y}^{t, x}, \underline{Z}^{t, x}\right)$ is a bounded solution to BSDE (4.1). By passing to the limit when $m \rightarrow \infty$ in (4.21), we derive (4.20) immediately.

\subsection{Bounded and Continuous Case}

In the smooth case, the dynamic utility function is a solution to BSDE (4.1) by Theorem 4.1. This remains true in more general case.

Proposition 4.4. Let us suppose that $\Phi$ is bounded and continuous, and $b$ and $\sigma$ satisfy the assumption $(\overline{4.17})$. Then there exists a bounded solution $\left(\bar{Y}^{t, x}, \bar{Z}^{t, x}\right)$ such that

$$
\bar{Y}_{s}^{t, x}=\inf \left\{E_{Q}\left[\Phi\left(X_{T}^{t, x}\right)+\int_{s}^{T} f\left(q_{r}\right) d r \mid \mathcal{F}_{s}\right] \mid Q \sim P\right\}
$$

Proof. By the same technique as that used in Proposition 4.3, let us define the function

$$
\bar{\Phi}_{m}(u)=\sup \left\{\Phi(p)-m|p-u|: p \in \mathbb{R}^{n}\right\}
$$

for each integer $m \geq 0$. Then $\bar{\Phi}_{m}$ is also bounded and globally Lipschitz with Lipschitz constant $m .\left(\bar{\Phi}_{m}\right)_{m \geq 0}$ is decreasing and converges pointwise to $\Phi$ with

$$
\|\Phi\|_{\infty} \geq \bar{\Phi}_{m} \geq \Phi
$$


Let $\left(\bar{Y}^{m ; t, x}, \bar{Z}^{m ; t, x}\right)$ be the solution to $\operatorname{BSDE}\left(g, \bar{\Phi}_{m}\left(X_{T}^{t, x}\right)\right)$. It follows from the same argument as that in Proposition 4.3 that by setting

$$
\bar{Y}^{t, x}=\lim _{m \rightarrow \infty} \bar{Y}^{m ; t, x}, \quad \quad \bar{Z}^{t, x}=\varlimsup_{m \rightarrow \infty} \bar{Z}^{m ; t, x},
$$

$\left(\bar{Y}^{t, x}, \bar{Z}^{t, x}\right)$ satisfies (4.23). On the other hand, since

$$
\underline{Y}_{s}^{m ; t, x} \leq \bar{Y}_{s}^{m ; t, x} \leq E\left[\bar{\Phi}_{m}\left(X_{T}^{t, x}\right) \mid \mathcal{F}_{s}\right]
$$

we deduce that

$$
\underline{Y}_{s}^{t, x} \leq \bar{Y}_{s}^{t, x} \leq E\left[\Phi\left(X_{T}^{t, x}\right) \mid \mathcal{F}_{s}\right]
$$

which implies that

$$
\lim _{s \rightarrow T} \bar{Y}_{s}^{t, x}=\Phi\left(X_{T}^{t, x}\right)
$$

Hence $\left(\bar{Y}^{t, x}, \bar{Z}^{t, x}\right)$ is also a bounded solution to $\operatorname{BSDE}\left(g, \Phi\left(X_{T}^{t, x}\right)\right)$. Lemma 2.3 implies that any bounded solution of BSDE is less than or equal to the corresponding dynamic utility function,

$$
\bar{Y}_{s}^{t, x} \leq \operatorname{ess.inf}\left\{E_{Q}\left[\Phi\left(X_{T}^{t, x}\right)+\int_{s}^{T} f\left(q_{r}\right) d r \mid \mathcal{F}_{s}\right] \mid Q \sim P\right\} .
$$

Finally, as

$$
\bar{Y}_{s}^{m ; t, x}=\operatorname{ess.inf}\left\{E_{Q}\left[\bar{\Phi}_{m}\left(X_{T}^{t, x}\right)+\int_{s}^{T} f\left(q_{r}\right) d r \mid \mathcal{F}_{s}\right] \mid Q \sim P\right\}
$$

and $\bar{\Phi}_{m}\left(X_{T}^{t, x}\right)$ converges decreasingly to $\Phi\left(X_{T}^{t, x}\right)$, we deduce

$$
\bar{Y}_{s}^{t, x} \geq \operatorname{ess.inf}\left\{E_{Q}\left[\Phi\left(X_{T}^{t, x}\right)+\int_{s}^{T} f\left(q_{r}\right) d r \mid \mathcal{F}_{s}\right] \mid Q \sim P\right\} .
$$

Combining the above, we conclude that the solution $\bar{Y}^{t, x}$ is a dynamic utility function.

Notice that we used $\underline{\Phi}^{m}$ to approximate $\Phi$ in the lower semi-continuous case. In the continuous case, we can show that both $\underline{Y}^{m ; t, x}$ and $\bar{Y}^{m ; t, x}$ converge to the same limit. Now first let us consider the uniformly continuous case.

Theorem 4.3. Suppose that $\Phi$ is bounded and uniformly continuous. We then have

$$
\bar{Y}_{s}^{t, x}=U_{s}\left(\Phi\left(X_{T}^{t, x}\right)\right)=\underline{Y}_{s}^{t, x} .
$$


Proof. It follows from the uniform continuity of $\Phi$ that both $\left(\bar{\Phi}_{m}\right)_{m \geq 0}$ and $\left(\underline{\Phi}_{m}\right)_{m \geq 0}$ converge to $\Phi$ with the norm $\|\cdot\|_{\infty}$.

Indeed, the uniform continuity of $\Phi$ implies that, for any $\epsilon>0$, there exists $\delta_{\epsilon}>0$, such that if $|p-u| \leq \delta(\epsilon)$, then

$$
|\Phi(p)-\Phi(u)| \leq \epsilon
$$

By the definition, we get

$$
\Phi(u)-\underline{\Phi}_{m}(u)=\sup \left\{\Phi(u)-\Phi(p)-m|p-u|: p \in \mathbb{R}^{n}\right\} .
$$

But we have

$$
\begin{aligned}
& \Phi(u)-\Phi(p)-m|p-u| \\
= & (\Phi(u)-\Phi(p)) 1_{\left\{|p-u|<\delta_{\epsilon}\right\}}+(\Phi(u)-\Phi(p)) 1_{\left\{|p-u| \geq \delta_{\epsilon}\right\}}-m|p-u| \\
\leq & \epsilon+2 \frac{\|\Phi\|_{\infty}}{\delta_{\epsilon}}|p-u|-m|p-u|
\end{aligned}
$$

from which we deduce that if $m>2 \frac{\|\Phi\|_{\infty}}{\delta_{\epsilon}}$, then

$$
0 \leq \Phi(u)-\underline{\Phi}_{m}(u) \leq \epsilon, \forall u \in \mathbb{R}^{n}
$$

hence,

$$
\lim _{m \rightarrow \infty}\left\|\Phi-\underline{\Phi}_{m}\right\|_{\infty}=0
$$

Combining with the convergence of a dynamic utility function and

$$
\underline{Y}_{s}^{m ; t, x}=\operatorname{ess.inf}\left\{E_{Q}\left[\underline{\Phi}_{m}\left(X_{T}^{t, x}\right)+\int_{s}^{T} f\left(q_{r}\right) d r \mid \mathcal{F}_{s}\right] \mid Q \sim P\right\}
$$

we get

$$
\underline{Y}_{s}^{t, x}=U_{s}\left(\Phi\left(X_{T}^{t, x}\right)\right)=\operatorname{ess.inf}\left\{E_{Q}\left[\Phi\left(X_{T}^{t, x}\right)+\int_{s}^{T} f\left(q_{r}\right) d r \mid \mathcal{F}_{s}\right] \mid Q \sim P\right\} .
$$

By the same argument or simply by Proposition 4.4, we have

$$
\bar{Y}_{s}^{t, x}=U_{s}\left(\Phi\left(X_{T}^{t, x}\right)\right)=\underline{Y}_{s}^{t, x}
$$

Let us now consider the general case: $\Phi$ is bounded and continuous. 
Theorem 4.4. Suppose that $\Phi$ is bounded and continuous and $f$ satisfies the assumption: there exists a constant $M$ such that

$$
\alpha:=\min _{|x|=M}\{f(x)\}>0
$$

We then have

$$
\bar{Y}_{s}^{t, x}=U_{s}\left(\Phi\left(X_{T}^{t, x}\right)\right)=\underline{Y}_{s}^{t, x}, \quad \forall s \in[0, T] .
$$

Proof. First, in the special case $X_{T}^{t, x}=x+B_{T}-B_{t}$, let us consider $\bar{Y}^{m ; t, x}-\underline{Y}^{m ; t, x}$. It follows from (4.2) that

$$
\begin{aligned}
& \bar{Y}_{0}^{m ; t, x}-\underline{Y}_{0}^{m ; t, x} \\
= & \operatorname{ess.inf}\left\{E_{Q}\left[\bar{\Phi}_{m}\left(X_{T}^{t, x}\right)+\int_{0}^{T} f\left(q_{u}\right) d u\right] \mid Q \sim P, E_{Q}\left[\int_{0}^{T} f\left(q_{u}\right) d u\right] \leq 2\|\Phi\|_{\infty}\right\} \\
& -\operatorname{ess.inf}\left\{E_{Q}\left[\underline{\Phi}_{m}\left(X_{T}^{t, x}\right)+\int_{0}^{T} f\left(q_{u}\right) d u\right] \mid Q \sim P, E_{Q}\left[\int_{0}^{T} f\left(q_{u}\right) d u\right] \leq 2\|\Phi\|_{\infty}\right\} \\
\leq & \operatorname{ess.sup}\left\{E_{Q}\left[\bar{\Phi}_{m}\left(X_{T}^{t, x}\right)-\underline{\Phi}_{m}\left(X_{T}^{t, x}\right)\right] \mid Q \sim P, E_{Q}\left[\int_{0}^{T} f\left(q_{u}\right) d u\right] \leq 2\|\Phi\|_{\infty}\right\} .
\end{aligned}
$$

Denoting $\bar{\Phi}_{m}-\underline{\Phi}_{m}$ as $\Psi_{m}$, then $\Psi_{m}$ is continuous. We then analyze

$$
\Psi_{m}\left(x+B_{T}-B_{t}\right)=\Psi_{m}\left(x+B_{T}^{Q}-B_{t}^{Q}+\int_{t}^{T} q_{u} d u\right)
$$

in three parts. First, we have, for $N>M$,

$$
\begin{aligned}
& E_{Q}\left[\Psi_{m}\left(x+B_{T}^{Q}-B_{t}^{Q}+\int_{t}^{T} q_{u} d u\right) 1_{\left\{\left|\int_{t}^{T} q_{u} d u\right|>N\right\}}\right] \\
\leq & 2\|\Phi\|_{\infty} Q\left(\left\{\left|\int_{t}^{T} q_{u} d u\right|>N\right\}\right) \\
\leq & 2\|\Phi\|_{\infty} \frac{E_{Q}\left[\left|\int_{t}^{T} q_{u} d u\right|\right]}{N} .
\end{aligned}
$$

Combining with

$$
\begin{aligned}
& E_{Q}\left[\left|\int_{t}^{T} q_{u} d u\right|\right] \\
\leq & E_{Q}\left[\int_{t}^{T}\left|q_{u}\right| 1_{\left\{\left|q_{u}\right|<M\right\}} d u\right]+E_{Q}\left[\int_{t}^{T}\left|q_{u}\right| 1_{\left\{\left|q_{u}\right| \geq M\right\}} d u\right] \\
\leq & M T+E_{Q}\left[\int_{0}^{T} \frac{M}{\alpha} f\left(q_{u}\right) 1_{\left\{\left|q_{u}\right| \geq M\right\}} d u\right] \\
\leq & M T+2 \frac{M}{\alpha}\|\Phi\|_{\infty},
\end{aligned}
$$

we deduce that

$$
E_{Q}\left[\Psi_{m}\left(x+B_{T}^{Q}-B_{t}^{Q}+\int_{t}^{T} q_{u} d u\right) 1_{\left\{\left|\int_{t}^{T} q_{u} d u\right|>N\right\}}\right] \leq \frac{c_{1}}{N},
$$


where $c_{1}>0$ is a constant. Second, we have

$$
\begin{aligned}
& E_{Q}\left[\Psi_{m}\left(x+B_{T}^{Q}-B_{t}^{Q}+\int_{t}^{T} q_{u} d u\right) 1_{\left\{\left|B_{T}^{Q}-B_{t}^{Q}\right|>N,\left|\int_{t}^{T} q_{u} d u\right| \leq N\right\}}\right] \\
\leq & 2\|\Phi\|_{\infty} \frac{E_{Q}\left[\left|B_{T}^{Q}-B_{t}^{Q}\right|\right]}{N} \\
= & \frac{c_{2}}{N}
\end{aligned}
$$

where $c_{2}>0$ is a constant independent of $Q$ and $m$. Third, we have

$$
\begin{aligned}
& E_{Q}\left[\Psi_{m}\left(x+B_{T}^{Q}-B_{t}^{Q}+\int_{t}^{T} q_{u} d u\right) 1_{\left\{\left|B_{T}^{Q}-B_{t}^{Q}\right| \leq N,\left|\int_{t}^{T} q_{u} d u\right| \leq N\right\}}\right] \\
& \leq \sup _{|y| \leq|x|+2 N} \Psi_{m}(y) \text {. }
\end{aligned}
$$

It follows from the preceding three estimates that

$$
E_{Q}\left[\Psi_{m}\left(x+B_{T}-B_{t}\right)\right] \leq \frac{c_{1}+c_{2}}{N}+\sup _{|y| \leq|x|+2 N} \Psi_{m}(y)
$$

which implies that

$$
\begin{aligned}
\varlimsup_{m \rightarrow \infty}\left(\bar{Y}_{0}^{m ; t, x}-\underline{Y}_{0}^{m ; t, x}\right) & \leq \varlimsup_{m \rightarrow \infty}\left[\frac{c_{1}+c_{2}}{N}+\sup _{|y| \leq|x|+2 N} \Psi_{m}(y)\right] \\
& =\frac{c_{1}+c_{2}}{N} .
\end{aligned}
$$

Since $\bar{Y}_{0}^{m ; t, x}-\underline{Y}_{0}^{m ; t, x} \geq 0$, by letting $N$ tend to $\infty$, we deduce that

$$
\bar{Y}_{0}^{t, x}=\underline{Y}_{0}^{t, x}
$$

Combining with the Markov property of $\bar{Y}^{t, x}$ and $\underline{Y}^{t, x}$, we conclude that $\bar{Y}_{s}^{t, x}=\underline{Y}_{s}^{t, x}$, for any $0<s<T$.

Notice that essentially we have made use of the simple fact that

$$
\lim _{m \rightarrow \infty} \sup _{|y| \leq c} \Psi_{m}(y)=0
$$

where $c$ is a constant. So in the general case,

$$
X_{s}^{t, x}=x+\int_{t}^{s} b\left(u, X_{u}^{t, x}\right) d u+\sigma\left(B_{s}^{Q}-B_{t}^{Q}+\int_{t}^{s} q_{u} d u\right),
$$

since $b$ is Lipschitz, applying Gronwall's inequality, we get

$$
\left|X_{T}^{t, x}\right| \leq \sup _{t \leq s \leq T}\left|X_{s}^{t, x}\right| \leq C\left(1+\int_{t}^{T}\left|q_{u}\right| d u+\sup _{t \leq s \leq T}\left|B_{s}^{Q}-B_{t}^{Q}\right|\right),
$$


where $C>0$ is a constant. Hence the same proof works.

Now we define

$$
\bar{u}^{m}(t, x):=\bar{Y}_{t}^{m ; t, x}, \underline{u}^{m}(t, x):=\underline{Y}_{t}^{m ; t, x}
$$

and

$$
u(t, x):=U_{t}\left(\Phi\left(X_{T}^{t, x}\right)\right)
$$

We have the following theorem.

Theorem 4.5. Suppose that $\Phi$ is bounded and continuous and $f$ satisfies the assumption: there exists a constant $M>0$ such that

$$
\alpha:=\min _{|x|=M}\{f(x)\}>0
$$

and that $b$ and $\sigma$ satisfy the assumption 4.17). Then $u(t, x)$ defined by (4.28) is a bounded and continuous deterministic function on $[0, T] \times \mathbb{R}^{n}$ and it is a viscosity solution to PDE (4.9).

Proof. Theorem 4.4 implies that $\left\{\bar{u}^{m}(t, x)\right\}_{m=1}^{\infty}$ (resp. $\left.\left\{\underline{u}^{m}(t, x)\right\}_{m=1}^{\infty}\right)$ converges decreasingly (resp. increasingly) to $u(t, x)$. Combining with the continuity of $\bar{u}^{m}(t, x)$ and $\underline{u}^{m}(t, x)$, we deduce that $u(t, x)$ is continuous.

By Dini's theorem, they converge to $u$ uniformly in any compact set. This implies that $u$ is a viscosity solution by the stability theorem of viscosity solutions to PDEs (see, e.g., [6]) .

Acknowledgments This research was sponsored by a grant of Credit Suisse as well as by a grant NCCR-Finrisk. The text only reflects the opinion of the authors. We also thank M. Ben-Artzi for stimulating discussions regarding the connection with viscous Hamilton-Jacobi equations.

\section{References}

[1] M. Ben-Artzi, P. Souplet and F. B. Weissler, The local theory for viscous Hamilton-Jacobi equations in Lebesgue spaces. J. Math. Pures Appl. 81 (2002), 343-378.

[2] J. M. Bismut, Conjugate convex functions in optimal stochastic control. J. Math. Anal. Appl. 44 (1973), 384-404. 
[3] P. Briand and Y. Hu, BSDE with quadratic growth and unbounded terminal value. Probab. Theory Related Fields 136 (2006), 604-618.

[4] P. Briand and Y. Hu, Quadratic BSDEs with convex generators and unbounded terminal conditions. Probab. Theory Related Fields 141 (2008), 543-567.

[5] P. Cheridito, H. M. Soner, N. Touzi and N. Victoir, Second-order backward stochastic differential equations and fully nonlinear parabolic PDEs. Comm. Pure Appl. Math. 60 (2007), 1081-1110.

[6] M. G. Crandall, H. Ishii and P. L. Lions, User's guide to viscosity solutions of second order partial differential equations. Bull. Amer. Math. Soc. 27 (1992), $1-67$.

[7] F. Delbaen, S. Peng and E. Rosazza Gianin, Representation of the penalty term of dynamic concave utilities. arXiv:0802.1121v1 [math.PR] 8 Feb 2008.

[8] N. El Karoui, S. Peng and M. C. Quenez, Backward stochastic differential equations in finance. Math. Finance 7 (1997), 1-71.

[9] H. Föllmer and A. Schied, Convex measures of risk and trading constraints. Finance Stoch. 6 (2002), 429-447.

[10] B. H. Gilding, M. Guedda and R. Kersner, The Cauchy problem for $u_{t}=\Delta u+$ $|\nabla u|^{q}$. J. Math. Anal. Appl. 284 (2003), 733-755.

[11] E. Jouini, W. Schachermayer and N. Touzi, Law invariant risk measures have the Fatou property. Advances in mathematical economics. 9, 49-71, Adv. Math. Econ., 9, Springer, Tokyo, 2006.

[12] M. Kobylanski, Backward stochastic differential equations and partial differential equations with quadratic growth. Ann. Probab. 28 (2000), 558-602.

[13] E. Pardoux and S. Peng, Adapted solution of a backward stochastic differential equation. Systems Control Lett. 14 (1990), 55-61.

[14] E. Pardoux and S. Peng, Backward stochastic differential equations and quasilinear parabolic partial differential equations. Stochastic partial differential equations and their applications (Charlotte, NC, 1991), 200-217, Lecture Notes in Control and Inform. Sci., 176, Springer, Berlin, 1992. 